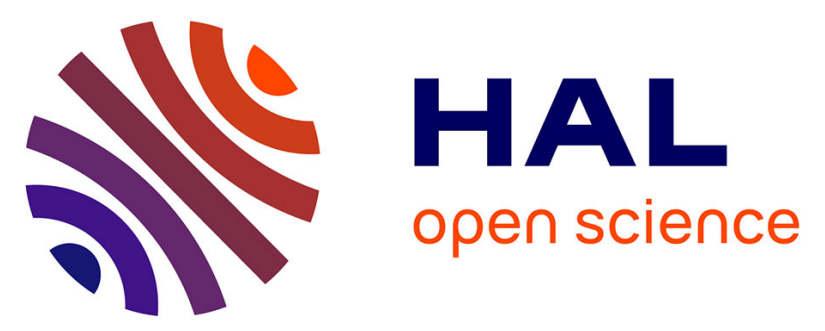

\title{
Toll-like receptor 7 agonist GS-9620 induces prolonged inhibition of HBV via a type I interferon-dependent mechanism
}

Congrong Niu, Li Li, Stephane Daffis, Julie Lucifora, Marc Bonnin, Sarah Maadadi, Eduardo Salas, Ruth Chu, Hilario Ramos, Christine Livingston, et al.

\section{To cite this version:}

Congrong Niu, Li Li, Stephane Daffis, Julie Lucifora, Marc Bonnin, et al.. Toll-like receptor 7 agonist GS-9620 induces prolonged inhibition of HBV via a type I interferon-dependent mechanism. Journal of Hepatology, 2018, 68 (5), pp.922-931. 10.1016/j.jhep.2017.12.007 hal-03315726

\author{
HAL Id: hal-03315726 \\ https://hal.science/hal-03315726
}

Submitted on 12 Aug 2021

HAL is a multi-disciplinary open access archive for the deposit and dissemination of scientific research documents, whether they are published or not. The documents may come from teaching and research institutions in France or abroad, or from public or private research centers.
L'archive ouverte pluridisciplinaire HAL, est destinée au dépôt et à la diffusion de documents scientifiques de niveau recherche, publiés ou non, émanant des établissements d'enseignement et de recherche français ou étrangers, des laboratoires publics ou privés. 


\section{Accepted Manuscript}

Toll-Like Receptor 7 Agonist GS-9620 Induces Prolonged Inhibition of HBV via a Type I Interferon-Dependent Mechanism

Congrong Niu, Li Li, Stephane Daffis, Julie Lucifora, Marc Bonnin, Sarah Maadadi, Eduardo Salas, Ruth Chu, Hilario Ramos, Christine M. Livingston, Rudolf K. Beran, Abhishek V. Garg, Scott Balsitis, David Durantel, Fabien Zoulim, William E. Delaney IV, Simon P. Fletcher

PII: S0168-8278(17)32489-3

DOI: https://doi.org/10.1016/j.jhep.2017.12.007

Reference: JHEPAT 6789

To appear in: Journal of Hepatology

Received Date: 9 May 2017

Revised Date: 17 November 2017

Accepted Date: 6 December 2017

Please cite this article as: Niu, C., Li, L., Daffis, S., Lucifora, J., Bonnin, M., Maadadi, S., Salas, E., Chu, R., Ramos, H., Livingston, C.M., Beran, R.K., Garg, A.V., Balsitis, S., Durantel, D., Zoulim, F., Delaney, W.E. IV, Fletcher, S.P., Toll-Like Receptor 7 Agonist GS-9620 Induces Prolonged Inhibition of HBV via a Type I InterferonDependent Mechanism, Journal of Hepatology (2017), doi: https://doi.org/10.1016/j.jhep.2017.12.007

This is a PDF file of an unedited manuscript that has been accepted for publication. As a service to our customers we are providing this early version of the manuscript. The manuscript will undergo copyediting, typesetting, and review of the resulting proof before it is published in its final form. Please note that during the production process errors may be discovered which could affect the content, and all legal disclaimers that apply to the journal pertain. 


\section{Toll-Like Receptor 7 Agonist GS-9620 Induces Prolonged Inhibition of HBV via a Type I Interferon-Dependent Mechanism}

Congrong $\mathrm{Niu}^{1}, \mathrm{Li} \mathrm{Li}^{1}$, Stephane Daffis ${ }^{1}$, Julie Lucifora ${ }^{2}$, Marc Bonnin ${ }^{2}$, Sarah Maadadi ${ }^{2}$, Eduardo Salas ${ }^{1}, \underline{\text { Ruth Chu }}^{1}$, Hilario Ramos ${ }^{1}$, Christine M. Livingston ${ }^{1}$, Rudolf K. Beran ${ }^{1}$, Abhishek V. Garg ${ }^{1}$, Scott Balsitis ${ }^{1}$, David Durantel ${ }^{2}$, Fabien Zoulim ${ }^{2,3,4}$, William E. Delaney IV ${ }^{1}$, Simon P. Fletcher ${ }^{1 \#}$

${ }^{1}$ Gilead Sciences, Inc., Foster City, CA, USA

${ }^{2}$ INSERM 1052, Université Claude Bernard Lyon 1, CNRS 5286, Centre Léon Bérard, Centre de Recherche en Cancérologie de Lyon, Lyon, 69003, France

${ }^{3}$ Hospices Civils de Lyon (HCL), 69002 Lyon, France

${ }^{4}$ Institut Universitaire de France (IUF), 75005 Paris, France

\section{\# Corresponding Author:}

Mailing address: Gilead Sciences, Inc., 333 Lakeside Drive, Foster City, CA 94404, USA

Phone: (650) 372-7663. Fax: (650) 522-5890. E-mail: simon.fletcher@gilead.com

Key Words: TLR7; GS-9620; Hepatitis B virus; interferon-alfa; cccDNA; interferonstimulated gene; MHC; immunoproteasome; APOBEC; Smc5/6. 
Abbreviations: APOBEC3, A3; cccDNA, covalently closed circular DNA; CHB, chronic hepatitis B; CM, conditioned media; Ctrl, control; DEG, differentially expressed gene; FDR, false discovery rate; $\mathrm{HBeAg}$, hepatitis $\mathrm{B}$ e antigen; $\mathrm{HBsAg}$, hepatitis $\mathrm{B}$ surface antigen; HBV, hepatitis B virus; IFN- $\alpha$, interferon-alpha; IFNAR, IFN- $\alpha / \beta$ receptor; IPA, Ingenuity Pathway Analysis; ISG, interferon-stimulated gene; $\underline{\mathrm{mAb}}$, monoclonal antibody; MHC, major histocompatibility complex; ND10, nuclear domain 10; PBMC, peripheral blood mononuclear cell; pDC, plasmacytoid dendritic cell; $\mathrm{PHH}$, primary human hepatocyte; pMHC, peptide/MHC class I complex; $\underline{\text { Smc5/6, structural }}$ maintenance of chromosome 5/6 complex; TCR, T cell receptor; TLR7, toll-like receptor 7; Tx, treatment.

Conflict of interest: C. Niu, L. Li, S. Daffis, E. Salas, R. Chu, H. Ramos, C. Livingston, R. Beran, A. Garg, S. Balsitis, W. Delaney and S. Fletcher are employees of Gilead Sciences, Inc. J. Lucifora, M. Bonnin, S. Maadadi, D. Durantel and F. Zoulim have nothing to declare.

Financial support: This study was sponsored by Gilead Sciences, Inc. INSERM U1052 also received institutional funding from INSERM, as well as external grants from ANRS, FRM (Foundation for medical research; DEQ20110421327), and DEVweCAN LABEX (ANR-10-LABX-0061) of the "Université de Lyon', within the program “Investissements d'Avenir'” (ANR-11-IDEX-0007) operated by the French National Research Agency (ANR). 
Author's Contributions: Conceived study: S. Fletcher. Participated in research design:

S. Daffis, H. Ramos, S. Fletcher, A. Garg, S. Balsitis, W. Delaney, J. Lucifora, D. Durantel, F. Zoulim. Provided supervision: S. Daffis, S. Balsitis, W. Delaney, S. Fletcher. Conducted experiments: C. Niu, S. Daffis, J. Lucifora, M. Bonnin, S. Maadadi, E. Salas,

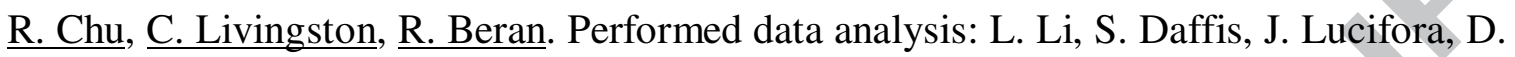
Durantel, S. Fletcher. Wrote or contributed to the writing of the manuscript: S. Daffis, D. Durantel, J. Lucifora, S. Fletcher. All authors reviewed and approved the final manuscript. 


\section{ABSTRACT}

Background \& Aims: GS-9620, an oral agonist of toll-like receptor 7 (TLR7), is in clinical development for the treatment of chronic hepatitis B (CHB). GS-9620 was previously shown to induce prolonged suppression of serum viral DNA and antigens in the woodchuck and chimpanzee models of $\mathrm{CHB}$. Here we investigated the molecular mechanisms that contribute to the antiviral response to GS-9620 using in vitro models of hepatitis B virus (HBV) infection. Methods: Cryopreserved primary human hepatocytes (PHH) and differentiated HepaRG (dHepaRG) cells were infected with HBV and treated with GS-9620, conditioned media from human peripheral blood mononuclear cells (PBMCs) treated with GS-9620 (GS-9620 conditioned media; GS-9620-CM), or other innate immune stimuli. The antiviral and transcriptional response to these agents was determined. Results: GS-9620 had no antiviral activity in HBV-infected PHH, consistent with low level TLR7 mRNA expression in human hepatocytes. In contrast, GS-9620-CM induced prolonged reduction of HBV DNA, RNA, and antigen levels in $\mathrm{PHH}$ and dHepaRG cells via a type I interferon (IFN)-dependent mechanism. GS-9620-CM did not reduce cccDNA levels in either cell type. Transcriptional profiling demonstrated that GS9620-CM strongly induced various HBV restriction factors - although not APOBEC3A or the Smc5/6 complex — and indicated that established HBV infection does not modulate innate immune sensing or signaling in cryopreserved PHH. GS-9620-CM also induced expression of immunoproteasome subunits and enhanced presentation of an immunodominant viral peptide in HBV-infected PHH. Conclusions: Type I IFN induced by GS-9620 durably suppressed HBV in human hepatocytes without reducing cccDNA levels. Moreover, $\mathrm{HBV}$ antigen presentation was enhanced, suggesting additional 
components of the TLR7-induced immune response played a role in the antiviral response to GS-9620 in animal models of $\mathrm{CHB}$.

Abstract word count: 272 (max: 275 words)

Lay Summary: GS-9620 is a drug in clinical trials for the treatment of chronic hepatitis B virus (HBV) infection. GS-9620 has previously been shown to suppress HBV in various animal models, but the underlying antiviral mechanisms were not completely understood. In this study, we determined that GS-9620 does not directly activate antiviral pathways in human liver cells, but can induce prolonged suppression of HBV via induction of an antiviral cytokine called interferon. However, interferon did not destroy the HBV genome, suggesting that other parts of the immune response (e.g. activation of immune cells that kill infected cells) also play an important role in the antiviral response to GS-9620. 


\section{INTRODUCTION}

Approximately 240 million individuals are chronically infected with hepatitis B virus (HBV), and over 650,000 people die each year due to $\mathrm{HBV}$-associated liver diseases, such as cirrhosis and hepatocellular carcinoma. Immunologic control of chronic hepatitis B (CHB), recognized as a "functional cure," is defined as sustained loss of HBV surface antigen (HBsAg) off therapy with or without seroconversion to anti-HBs antibody. Current therapies for $\mathrm{CHB}$ are limited to nucleos(t)ides and interferon-alpha (IFN- $\alpha$ ). These agents reduce viral load and improve long-term outcome, they rarely lead to cure. There is therefore an urgent need for new therapies that induce durable immune control of chronic HBV infection.

GS-9620 is a potent, orally bioavailable small molecule agonist of toll-like receptor 7 (TLR7) in clinical development for the treatment of CHB [1]. TLR7 is expressed in a subset of human immune cells, primarily plasmacytoid dendritic cells (pDCs) and B cells, and recognizes single-stranded RNA as well as small molecule agonists [2]. TLR7 activation induces innate and adaptive immune responses via induction of various cytokines (including multiple IFN- $\alpha$ subtypes) and chemokines, direct activation of B cells, and cross-priming of cytotoxic $\mathrm{CD}^{+} \mathrm{T}$ cells [3-5]. GS-9620 was previously shown to induce prolonged suppression of serum viral DNA and antigens in the chimpanzee and woodchuck models of CHB $[6,7]$. Various immunomodulatory activities may account for the antiviral effects of GS-9620 in these animal models (e.g., induction of antiviral cytokines, activation of $\mathrm{NK}$ cells, $\mathrm{CD}^{+} \mathrm{T}$ cells and $\mathrm{B}$ cells); however the exact mechanism remains unclear. Defining the molecular basis for response is an important 
goal because mechanistic understanding of GS-9620 activity could guide rational design of novel immunotherapeutic strategies.

In the current study we investigated the molecular mechanisms responsible for the antiviral response to GS-9620 using in vitro models of HBV infection in cryopreserved primary human hepatocytes $(\mathrm{PHH})$ as well as differentiated HepaRG (dHepaRG) cells. 


\section{MATERIALS AND METHODS}

\section{Ethics statement}

Consent was obtained from the donor or the donor's legal next of kin for use of $\mathrm{PHH}$, buffy coats from healthy human volunteers and whole blood from healthy human volunteers and $\mathrm{CHB}$ patients for research purposes using IRB-approved authorizations. Additional details are provided in the Supplementary Materials and the Supplementary CTAT Table.

\section{$\underline{\text { Additional materials and methods }}$}

Chemical and reagent details are provided in the Supplementary Materials and the Supplementary CTAT Table. Methods for production and characterization of conditioned media, infection and treatment of PHH and dHepaRG cells, HBeAg and HBsAg analysis, quantitative RT-PCR (qRT-PCR), HBV DNA analysis, cell viability analysis, HBV cccDNA analysis, epifluorescence microscopy, confocal microscopy, Western blotting, pMHC complex analysis and RNA-Seq are provided in the Supplementary Materials.

\section{Statistical analysis}

Data is expressed as mean \pm standard error of the mean (SEM). Statistical significance was tested using a two-tailed t-test (for two sample comparisons) or either one-way or two-way ANOVA with multiple comparison correction (for multiple comparisons). A value of $p<0.05$ was considered significant. 


\section{RESULTS}

Cytokines induced by GS-9620 reduce HBV DNA, RNA and antigens, but do not

\section{alter cccDNA levels}

We first determined that GS-9620 has no antiviral activity in $\mathrm{HBV}$-infected PHH, consistent with low level TLR7 mRNA expression in human hepatocytes (Supplementary Figs.1-3). These data are described in the Supplementary Materials. We next evaluated whether cytokines induced by GS-9620 in human PBMCs can suppress HBV after infection of PHH and dHepaRG cells. For these studies, PBMCs were stimulated with 10 nM GS-9620 since this concentration strongly induces IFN- $\alpha$, a prototypic TLR7 cytokine with antiviral activities (Supplementary Fig. 4A). HBV-infected PHH were continuously treated (day 3-20 post-infection) with IFN- $\alpha$ or with conditioned media from PBMCs stimulated with GS-9620 (GS-9620-CM) or DMSO (Mock-CM). Treatment with both GS-9620-CM and 100 IU/mL IFN- $\alpha$ strongly reduced the levels of HBV DNA and HBeAg (>75\%), as well as HBV RNA (>70\%) and HBsAg (>60\%), with only a very modest effect ( $<3 \%$ reduction) on cell viability (Figs. 1A-E, Supplementary Fig. 2B). GS-9620-CM and IFN- $\alpha$ also had antiviral activity in HBV-infected PHH when treatment was delayed (day 13-20 post-infection) (Figs. 1A-D). PHH viability was not affected by this shorter treatment duration (Fig. 1E, Supplementary Fig. 2B). GS-9620$\mathrm{CM}$ also had antiviral activity in HBV-infected dHepaRG cells, although the HBsAg reduction was relatively modest (30\%) (Fig. 1F). Importantly, Mock-CM did not have antiviral activity in either HBV-infected PHH or dHepaRG cells (Fig. 1). 
In contrast to the other viral parameters, GS-9620-CM treatment did not alter cccDNA levels in HBV-infected PHH or dHepaRG cells (Fig. 2). However, even though cccDNA levels were not changed by GS-9620-CM and IFN- $\alpha$, all other viral endpoints were still significantly reduced 7 days after cessation of treatment (day 3-13 post-infection) of HBV-infected PHH (Figs. 1A-D). Similarly, HBV DNA levels were significantly reduced 11 days after cessation of GS-9620-CM treatment of HBV-infected dHepaRG cells, whereas viral DNA rebounded to control levels after tenofovir treatment was stopped (Supplementary Fig. 5A). A longer duration study in HBV-infected dHepaRG cells revealed that HBV DNA and HBsAg levels were still significantly suppressed 35 days after cessation of GS-9620-CM treatment (Supplementary Fig. 6). Collectively, these data demonstrate that GS-9620-CM can induce a prolonged antiviral effect in HBVinfected PHH and dHepaRG cells without reducing cccDNA levels. In contrast to IFN- $\alpha$, TNF- $\alpha$ did not induce prolonged suppression of viral RNA in HBV-infected PHH once treatment was stopped (Supplementary Fig. 5B). This suggests important mechanistic differences underlie the antiviral response to these cytokines.

\section{GS-9620-induced cytokines inhibit HBV via a type I IFN-dependent mechanism}

We next characterized GS-9620-CM by luminex analysis to identify the cytokine(s) responsible for the antiviral activity observed in HBV-infected PHH and dHepaRG cells. As expected, IFN- $\alpha$ was the predominant cytokine in GS-9620-CM, although lower amounts of other cytokines (e.g. IFN- $\gamma$, IL-1 $\beta$, IL-6 and TNF- $\alpha$ ) with antiviral activity against HBV were also present (Fig. 3A) [8]. IFN- $\beta$, like IFN- $\alpha$, is a type I IFN that signals via the IFN- $\alpha / \beta$ receptor (IFNAR). In contrast to IFN- $\alpha$, there were very low 
levels of IFN- $\beta$ in GS-9620-CM $(<0.5 \mathrm{pg} / \mathrm{mL})$. Importantly, the antiviral cytokine profile of GS-9620-CM was similar to the serum cytokine profile in chimpanzees dosed orally with GS-9620 [6]. Moreover, GS-9620 induced comparable IFN- $\alpha$ levels in vitro in human PBMCs from healthy donors and CHB patients (Supplementary Fig. 4B). Collectively, these data support the use of GS-9620-CM from healthy human donor PBMCs in these studies

In line with the PBMC cytokine profile, antibody blockade of the type I IFN receptor (IFNAR) or knock-down of IFNAR1 by RNA interference abrogated the antiviral activity of GS-9620-CM in HBV-infected PHH (Fig. 3B-E). The antiviral activity of GS-9620CM in HBV-infected dHepaRG cells was also blocked by an anti-IFNAR antibody, but not by TNF- $\alpha$ or IFN- $\gamma$ neutralizing antibodies (Supplementary Fig. 7). Taken together, these data indicate that type I IFN (most likely IFN- $\alpha$ ) is the cytokine responsible for the HBV suppressive activity of GS-9620-CM.

\section{Established HBV infection does not significantly modulate the transcriptional response to GS-9620-induced cytokines}

A recent study suggested that $\mathrm{HBV}$ can inhibit IFN- $\alpha$ signaling in a humanized mouse model [9]. Since GS-9620-CM is principally composed of IFN- $\alpha$ and mediates its antiviral effect via the type I IFN receptor, we evaluated whether HBV suppresses the global transcriptional response to GS-9620-CM in PHH. The experimental set-up was designed to evaluate the potential impact of established HBV infection on the hepatocyte innate immune response, since this scenario is clinically relevant to the treatment of CHB 
(Fig. 4A). High-level infection ( 80-90\% PHH infected) was confirmed by HBV core antigen staining (Fig. 4B). GS-9620-CM induced the greatest transcriptional response 8 hours post-treatment, with $>1000$ genes being differentially expressed (FDR $<0.05$, foldchange >2) in each PHH donor (Fig. 4C, Supplementary Table 5). Interestingly, the number of differentially expressed genes (DEGs) and the overall transcriptional profile were comparable in mock-infected and $\mathrm{HBV}$-infected $\mathrm{PHH}$, as well as in different $\mathrm{PHH}$ donors (Fig. 4C-D).

Ingenuity Pathway Analysis (IPA) and Gene Set Enrichment Analysis (GSEA) both identified IFN signaling as the top transcriptional pathway induced by GS-9620-CM in HBV-infected PHH (Fig. 5A, Supplementary Table 1). In line with the whole transcriptome analyses, GS-9620-CM induced a comparable interferon-stimulated gene (ISG) response in mock-infected and $\mathrm{HBV}$-infected $\mathrm{PHH}$, with no ISG being differentially modulated by GS-9620-CM in mock-infected vs. HBV-infected $\mathrm{PHH}$ in both donors (Figs. 5B and C, Supplementary Table 7). Taken together, these data suggest that established HBV infection does not modulate the transcriptional response to IFN- $\alpha$ in our cell culture system.

\section{Established HBV infection does not significantly modulate the transcriptional}

\section{response to various innate immune stimuli}

In light of the GS-9620-CM transcriptome analysis, we next evaluated whether the PHH response to diverse innate immune stimuli is influenced by established HBV infection (Fig. 6A). In addition to GS-9620-CM and IFN- $\alpha$, this study evaluated the ISG response 
to poly(I:C) and Sendai virus $(\mathrm{SeV})$, which are agonists of TLR3/MDA5 and RIG-I, respectively. The transcriptional response was measured by qRT-PCR at 8 hours posttreatment since this was the peak (or close to the peak) of the response in PHH to various innate immune stimuli in previous studies (Fig. 5B) [10]. High-level infection ( 80-90\% $\mathrm{PHH}$ infected) at the time of innate immune stimulation was confirmed by HBV core antigen staining (Fig. 6B). Consistent with the GS-9620-CM RNA-Seq data, HBV infection did not significantly reduce the ISG response to any of the innate immune stimuli tested (Fig. 6C, Supplementary Fig. 8). This was the case whether the magnitude of ISG induction was high (e.g. RSAD2 (viperin), >100-fold induction) or low (e.g. EIF2AK2 (PKR), <10-fold induction). Of note, although not statistically significant, there was a trend towards lower ISG induction in HBV-infected PHH for certain ISGs. However, this equated to only a $10 \pm 3 \%$ (mean \pm SEM) difference in fold-change for the multiple genes evaluated $(n=8)$ in three different $\mathrm{PHH}$ donors with various innate immune stimuli. This lack of significant, coordinated ISG repression suggests that established HBV infection does not interfere with the hepatocyte transcriptional response to IFN- $\alpha$ or TLR3/MDA5 and RIG-I agonists in our cell culture system.

GS-9620-induced cytokines strongly increase the expression of various HBV restriction factors, but not APOBEC3A or the Smc5/6 complex

GS-9620-CM induced the expression of a large number of ISGs in PHH (Fig. 5B, Supplementary Table 6), many of which have antiviral effector functions [11]. The ISG expression profile in HBV-infected PHH treated with GS-9620-CM was similar to the intrahepatic ISG signature in CHB chimpanzees treated with GS-9620, although a subset 
of ISGs (including several chemokines) was differentially induced in the two systems (Supplementary Fig. 9). Importantly, none of the ISGs that were strongly induced in PHH but not chimpanzee liver (i.e. ANGPTL1, BCL2L14 and CXCL9) have been reported to possess direct antiviral effector function. This suggests that the differential induction of these genes does not impact the utility of the in vitro PHH model for investigating the molecular mechanisms responsible for the antiviral response to GS-9620 in vivo.

The HBV restriction factors $M x A(M X 1)$ and tetherin (BST2) were amongst the ISGs induced by GS-9620-CM in HBV-infected PHH (Supplementary Table 6). However, MxA and tetherin do not reduce total HBV RNA levels [12, 13], suggesting that other HBV restriction factors were also induced by GS-9620-CM. We therefore evaluated expression of APOBEC3 proteins, which have been reported to mediate cccDNA deamination and degradation $[14,15]$, as well as the structural maintenance of chromosome 5/6 complex (Smc5/6), which suppresses cccDNA transcription when localized to intranuclear structures known as nuclear domain 10 (ND10) [10, 16].

We determined that GS-9620-CM and IFN- $\alpha$ treatment induced expression of the ND10 components PML and SP100, but not Smc5/6 subunits (Supplementary Table 2, Supplementary Fig. 10A and B). Interestingly, IFN- $\alpha$ altered the nuclear distribution of Smc6 by significantly increasing the number of ND10 (i.e. PML foci) in uninfected PHH (Supplementary Fig. 10C and D). However, since Smc5/6 is effectively degraded in HBV-infected PHH and is not induced by IFN- $\alpha$, suppression of HBV RNA by GS9620-CM is likely independent of this restriction factor. 
In contrast to Smc5/6, APOBEC3B (A3B) was modestly induced by GS-9620-CM (Supplementary Table 3). However, A3A expression was not increased by GS-9620induced cytokines in these PHH donors (Supplementary Table 3). Analysis of additional PHH identified a donor in which $A 3 A$ expression was significantly induced by GS-9620$\mathrm{CM}$ as well other innate immune stimuli (Supplementary Fig. 11); however, $A 3 A$ induction by GS-9620-CM treatment of this donor was substantially lower than observed with IFN- $\alpha$ treatment of freshly isolated PHH in a previous study [14]. Consistent with the antiviral response in the other PHH, GS-9620-CM did not reduce cccDNA levels in

this donor (Supplementary Fig. 12, PHH donor 1). Treatment with a high concentration of IFN- $\alpha(1000 \mathrm{IU} / \mathrm{mL})$ also did not reduce cccDNA levels in this or other PHH donors (Supplementary Fig. 12). Taken together, these data demonstrate that GS-9620-CM did not strongly increase the expression of $A 3 A$ and $A 3 B$ and did not induce cccDNA degradation in our cell culture system.

Cytokines induced by GS-9620 enhance presentation of an immunodominant HBV peptide

Antigen presentation by human hepatocytes is thought to be generally inefficient [17]. It was therefore interesting that transcriptional pathway analysis revealed GS-9620-CM induced antigen processing and presentation signatures in HBV-infected PHH (Fig. 5A, Supplementary Table 1). In line with the pathway analysis, GS-9620-CM induced expression of immunoproteasome subunits (PSMB8, PSMB9 and PSMB10) as well as various MHC class I antigen presentation genes (e.g. TAP1,TAP2, HLA genes) and a 
regulator of class I MHC expression (NLRC5) in PHH (Supplementary Table 4). In contrast, constitutive proteasome subunits (PSMB5, PSMB6 and PSMB7) were not identified as GS-9620-CM DEGs (Supplementary Table 4). Consistent with the analysis of other ISGs, induction of these antigen processing and presentation genes was comparable in mock-infected and HBV-infected PHH (Supplementary Table 7).

A previous study determined that immunoproteasome subunits do not play a role in IFNmediation inhibition of viral replication in HBV transgenic mice, but influence both the magnitude and specificity of the $\mathrm{CD}^{+} \mathrm{T}$ cells response to HBV proteins [18]. Since presentation of the immunodominant HBsAg 183-191 (Env183-191) peptide by MHC class I is enhanced by expression of immunoproteasome subunits in transgenic mice [18], we used an antibody which recognizes the Env183-191 peptide:HLA-A0201 (pMHC) complex [19] to determine whether GS-9620-CM influences presentation of this peptide by HBV-infected PHH. This monoclonal antibody (Env183-191 mAb) was validated by demonstrating specific binding to the cognate pMHC in peptide-pulsed T2 cells (Fig. 7A and B). Using the Env183-191 mAb, we determined that GS-9620-CM significantly induced presentation of Env183-191 by HBV-infected HLA-A0201 ${ }^{+}$PHH (Fig. 7C). Notably, this immunodominant viral peptide was not detected on HBV-infected PHH in the absence of IFN- $\gamma$ or GS-9620-CM treatment (Fig. 7C), in line with previous data indicating that this peptide is most efficiently processed by the immunoproteasome [18]. Together, these data suggest that GS-9620-induced cytokines can modulate HBV peptide presentation in $\mathrm{HBV}$-infected $\mathrm{PHH}$. 


\section{DISCUSSION}

The oral TLR7 agonist GS-9620 induced prolonged suppression of serum viral DNA and antigens in both the woodchuck and chimpanzee models of CHB [6, 7]. In the current study, we characterized the response of HBV-infected human hepatocytes to GS-9620 and GS-9620-induced cytokines to provide insights into the molecular mechanisms that contribute to the antiviral response in vivo. We demonstrated that GS-9620 has no direct antiviral activity in $\mathrm{HBV}$-infected $\mathrm{PHH}$, consistent with the low level TLR7 mRNA expression in human hepatocytes. In contrast, type I IFN — most likely IFN- $\alpha$ - induced by GS-9620 in PBMCs suppressed HBV RNA, DNA and antigens in HBV-infected PHH and dHepaRG cells, although it did not reduce cccDNA levels. Importantly, this antiviral response to GS-9620-induced IFN- $\alpha$ is consistent with previous studies with recombinant IFN- $\alpha$ in PHH as well as a humanized mouse model $[8,20]$. Our data are also in line with a previous study demonstrating type I IFN-dependent inhibition of HBV by a TLR7 agonist in a transgenic mouse model [21].

It has previously been reported that treatment with high dose recombinant IFN- $\alpha$ (1000 $\mathrm{U} / \mathrm{mL}$ ) triggers non-cytolytic degradation of cccDNA in human hepatocytes by APOBEC3 deaminases [14]. The current study was primarily focused on characterizing the antiviral response to lower doses of IFN- $\alpha$, either in GS-9620-CM (mean: $35 \mathrm{pg} / \mathrm{mL}$ )

or as recombinant IFN- $\alpha(100 \mathrm{IU} / \mathrm{mL})$. These lower amounts of IFN- $\alpha$ were evaluated because they were comparable to the serum IFN- $\alpha$ levels (mean: $119 \mathrm{pg} / \mathrm{mL}$ ) in $\mathrm{CHB}$ chimpanzees at the GS-9620 dose $(1 \mathrm{mg} / \mathrm{kg})$ that induced intrahepatic ISGs without modulating $\mathrm{CD}^{+} \mathrm{T}$ and $\mathrm{B}$ cells responses $[6,22]$. Importantly, the ISG expression profile 
in HBV-infected PHH treated with GS-9620-induced IFN- $\alpha$ was similar to the intrahepatic ISG signature in CHB chimpanzees treated with GS-9620, indicating these cytokine levels are translationally relevant. Nonetheless, it is important to note that, in contrast to the aforementioned study, high dose recombinant IFN- $\alpha(1000 \mathrm{IU} / \mathrm{mL}) \mathrm{did}$ not reduce cccDNA levels in our cryopreserved PHH. However, APOBEC 3A (A3A) expression was not induced by GS-9620-CM in most of our PHH donors, while $A 3 B$ expression was only modestly increased. Moreover, even in a PHH donor in which $A 3 A$ expression was increased by GS-9620-CM treatment, the magnitude of induction was lower than observed with IFN- $\alpha$ treatment of freshly isolated PHH in the previous study [14]. This suggests that there may be differential effects of IFN- $\alpha$ on ISG expression (and hence cccDNA stability) in cryopreserved vs. freshly isolated PHH. Differences in the cccDNA qPCR quantitation protocols (e.g. DNA extraction method, T5 exonuclease treatment, primers/probes and HBV standards) may also have contributed to the discrepancy between the studies. Accordingly, once the ongoing efforts to harmonize cccDNA quantification methods have been completed, it will be important to perform additional studies (e.g. in hepatocytes freshly isolated from CHB patients) to further investigate APOBEC3-mediated cccDNA degradation.

Identification of the key molecular effectors of HBV suppression by IFN- $\alpha$ has been challenging. The scale of this task is illustrated by the fact that GS-9620-CM strongly induced the expression of hundreds of ISGs in HBV-infected $\mathrm{PHH}$, including various well-characterized HBV restriction factors (e.g. MxA, tetherin) $[12,13]$. Additional layers of complexity are that intracellular localization (and not just expression level) can be 
important for the antiviral activity of HBV restriction factors $[10]$ and that IFN- $\alpha$ treatment can induce epigenetic modification of cccDNA [23, 24]. Indeed, epigenetic regulation of cccDNA may underlie the prolonged effect of GS-9620-CM treatment in our cell culture system. Interestingly, the epigenetic state of cccDNA in the absence of HBx (i.e. when the viral genome is transcriptionally repressed by Smc5/6), is similar to that induced by IFN- $\alpha$ treatment [25]. However, the lack of Smc5/6 induction by GS9620-CM is consistent with this complex being an intrinsic antiviral restriction factor [10] and suggests it does not play a role in type I IFN-mediated HBV suppression. However, if IFN- $\alpha$ reduces HBx protein levels and leads to reappearance of Smc5/6 in HBV-infected PHH, then this complex may play a role in maintaining cccDNA transcriptional suppression after cessation of treatment. Unfortunately, it is challenging to analyze HBx in HBV-infected PHH due to the lack of an antibody suitable for imaging studies.

The prolonged antiviral effect observed in vitro with GS-9620-CM may contribute to the sustained antiviral response induced by GS-9620 in animal models of CHB. However, the lack of cccDNA reduction by GS-9620-induced cytokines in our cell culture model suggests that additional components of the TLR7-induced immune response may have contributed to the antiviral response to GS-9620 in vivo. This interpretation is in line with recent transcriptomic and immunohistochemical analyses of liver tissue from the GS9260 chimpanzee study, which suggests that cytolytic CD $8^{+} \mathrm{T}$ cell effector mechanisms played a key role in the antiviral response to treatment [22]. Consequently, it is interesting that GS-9620-CM induced expression of antigen processing and presentation 
genes (including immunoproteasome subunits) and enhanced presentation of an immunodominant viral peptide in HBV-infected PHH. Intrahepatic expression of many of the same genes was induced during clearance of acute HBV infection in chimpanzees [26] and during the peak of antiviral response in GS-9620-treated chimpanzees [22]. This raises the possibility that GS-9620 may modulate the $\mathrm{CD}^{+} \mathrm{T}$ cell response to infected cells by altering presentation of HBV epitopes. However, GS-9620-CM also strongly induced $P D-L 1$ ( $C D 274$ ) expression in HBV-infected hepatocytes (Supplementary Table 4) and reduced viral protein levels within infected cells, both of which would be expected to influence the antiviral function of virus-specific $\mathrm{CD}^{+}$T cells $[17,27]$. GS-9620 may also impact the antiviral function of $\mathrm{HBV}$-specific $\mathrm{CD} 8^{+} \mathrm{T}$ cells by other mechanisms, for example by inducing immune cell migration, interactions and/or survival (as discussed in reference [22]), or by reducing HBV-mediated suppression of antiviral immunity by lowering HBV antigen levels. Further studies are required to evaluate these various hypotheses.

Another notable finding from this study is that established HBV infection does not inhibit the innate immune response of human hepatocytes in our cell culture model. Taken together with recent studies demonstrating that $\mathrm{HBV}$ infection does not induce a detectable innate immune response in $\mathrm{PHH}$, these data suggest that $\mathrm{HBV}$ may passively evade innate immune detection in hepatocytes $[10,28]$. These in vitro observations are in line with acute infection studies in chimpanzees, woodchucks and humans, as well as with the recently described STING deficiency of PHH [26, 29-31]. However, it is important to note that the levels of innate immune stimulation used in this study may 
have been sufficient to breach modest HBV-mediated inhibition of innate immune signaling $[9,20]$. In such a scenario, it is envisaged that HBV actively suppresses the (presumably low level) innate immune response induced by viral infection, but cannot counter the strong response induced by IFN- $\alpha$ or the innate immune activators used in this study. Additionally, our study did not examine whether HBV inhibits innate immune responses in hepatocytes early after infection, as has previously been reported [32]. These differences in experimental set-up may explain the discrepancy between our data and previous studies which have reported inhibition of hepatocyte innate immunity by HBV.

It is also possible that genetic and/or environmental factors influenced the $\mathrm{PHH}$ used in this study so that they did not accurately model the hepatocyte innate immune response in vivo. However, this is considered unlikely for several reasons. Firstly, the PHH were isolated from healthy donor livers and were selected based on the ability of the cells to plate and maintain high viability in culture. Mirroring the clinical situation when infected early in life, we have found that PHH from the vast majority of donors evaluated to-date can be efficiently infected when plating and viability conditions are achieved. Secondly, all PHH donors strongly responded to diverse innate immune stimuli. Thirdly, the wide age range of $\mathrm{PHH}$ donors used in this study $(3,8,28$ and 66 years old) would be expected to mitigate any potential role of environmental factors in the hepatocyte innate immune response. Nevertheless, it is very challenging to experimentally model chronic HBV infection in vitro. Effective in vitro modeling is further complicated by potential differences in the innate immune response and/or viral replication capacity of freshly isolated vs cryopreserved hepatocytes. Therefore, studies in hepatocytes freshly isolated 
from $\mathrm{CHB}$ patients will be required to confirm the validity of our findings using cryopreserved PHH from healthy donor livers.

In summary, our data provide important insights into the mechanisms underlying the antiviral response to GS-9620 in animal models of CHB. In addition, this study suggests that established HBV infection does not actively inhibit innate immunity in our cell culture model. This has important implications for how HBV establishes chronicity, as well as for the therapeutic response to innate immune agonists such as GS-9620. 


\section{ACKNOWLEDGEMENTS}

The authors gratefully acknowledge Daniel Tumas, Peng Yue, Aaron Avery, Anuj Gaggar, Tomas Cihlar and Frank Chisari for discussions and support; Mei Yu, Guofeng Cheng, Serge Karpinski and Dmytro Kornyeyev for technical assistance; Becky Norquist for manuscript preparation. 


\section{REFERENCES}

[1] Roethle PA, McFadden RM, Yang H, Hrvatin P, Hui H, Graupe M, et al. Identification and optimization of pteridinone Toll-like receptor 7 (TLR7) agonists for the oral treatment of viral hepatitis. J Med Chem 2013;56:7324-7333.

[2] Akira S, Takeda K. Toll-like receptor signalling. Nat Rev Immunol 2004;4:499511.

[3] Birmachu W, Gleason RM, Bulbulian BJ, Riter CL, Vasilakos JP, Lipson KE, et al. Transcriptional networks in plasmacytoid dendritic cells stimulated with synthetic TLR 7 agonists. BMC Immunol 2007;8:26.

[4] Hanten JA, Vasilakos JP, Riter CL, Neys L, Lipson KE, Alkan SS, et al. Comparison of human B cell activation by TLR7 and TLR9 agonists. BMC Immunol 2008;9:39.

[5] Oh JZ, Kurche JS, Burchill MA, Kedl RM. TLR7 enables cross-presentation by multiple dendritic cell subsets through a type I IFN-dependent pathway. Blood 2011;118:3028-3038.

[6] Lanford RE, Guerra B, Chavez D, Giavedoni L, Hodara VL, Brasky KM, et al. GS-9620, an oral agonist of Toll-like receptor-7, induces prolonged suppression of hepatitis B virus in chronically infected chimpanzees. Gastroenterology 2013;144:15081517.

[7] Menne S, Tumas DB, Liu KH, Thampi L, AlDeghaither D, Baldwin BH, et al. Sustained efficacy and seroconversion with the Toll-like receptor 7 agonist GS-9620 in the woodchuck model of chronic hepatitis B. J Hepatol 2015;62:1237-1245.

[8] Isorce N, Testoni B, Locatelli M, Fresquet J, Rivoire M, Luangsay S, et al. Antiviral activity of various interferons and pro-inflammatory cytokines in nontransformed cultured hepatocytes infected with hepatitis B virus. Antiviral Res 2016;130:36-45.

[9] Lutgehetmann M, Bornscheuer T, Volz T, Allweiss L, Bockmann JH, Pollok JM, et al. Hepatitis B virus limits response of human hepatocytes to interferon-alpha in chimeric mice. Gastroenterology 2011;140:2074-2083.

[10] Niu C, Livingston CM, Li L, Beran RK, Daffis S, Ramakrishnan D, et al. The Smc5/6 Complex Restricts HBV when Localized to ND10 without Inducing an Innate Immune Response and Is Counteracted by the HBV X Protein Shortly after Infection. PLoS One 2017;12:e0169648.

[11] Schoggins JW, Wilson SJ, Panis M, Murphy MY, Jones CT, Bieniasz P, et al. A diverse range of gene products are effectors of the type I interferon antiviral response. Nature 2011;472:481-485. 
[12] Li N, Zhang L, Chen L, Feng W, Xu Y, Chen F, et al. MxA inhibits hepatitis B virus replication by interaction with hepatitis B core antigen. Hepatology 2012;56:803811.

[13] Yan R, Zhao X, Cai D, Liu Y, Block T, Guo JT, et al. Interferon-inducible Protein Tetherin Inhibits Hepatitis B Virus Virion Secretion. J Virol 2015;89:9200-9212.

[14] Lucifora J, Xia Y, Reisinger F, Zhang K, Stadler D, Cheng X, et al. Specific and nonhepatotoxic degradation of nuclear hepatitis $B$ virus cccDNA. Science 2014;343:1221-1228.

[15] Xia Y, Stadler D, Lucifora J, Reisinger F, Webb D, Hosel M, et al. Interferongamma and Tumor Necrosis Factor-alpha Produced by $T$ Cells Reduce the HBV Persistence Form, cccDNA, Without Cytolysis. Gastroenterology 2016;150:194-205.

[16] Decorsière A, Mueller H, van Breugel PC, Abdul F, Gerossier L, Beran RK, et al. Hepatitis B virus $\mathrm{X}$ protein identifies the Smc5/6 complex as a host restriction factor. Nature 2016;531:386-389.

[17] Gehring AJ, Sun D, Kennedy PT, Nolte-'t Hoen E, Lim SG, Wasser S, et al. The level of viral antigen presented by hepatocytes influences CD8 T-cell function. J Virol 2007;81:2940-2949.

[18] Robek MD, Garcia ML, Boyd BS, Chisari FV. Role of immunoproteasome catalytic subunits in the immune response to hepatitis B virus. J Virol 2007;81:483-491.

[19] Sastry KS, Too CT, Kaur K, Gehring AJ, Low L, Javiad A, et al. Targeting hepatitis B virus-infected cells with a T-cell receptor-like antibody. J Virol 2011;85:1935-1942.

[20] Allweiss L, Volz T, Lutgehetmann M, Giersch K, Bornscheuer T, Lohse AW, et al. Immune cell responses are not required to induce substantial hepatitis B virus antigen decline during pegylated interferon-alpha administration. J Hepatol 2014;60:500-507.

[21] Isogawa M, Robek MD, Furuichi Y, Chisari FV. Toll-like receptor signaling inhibits hepatitis B virus replication in vivo. J Virol 2005;79:7269-7272.

[22] Li L, Barry V, Daffis S, Niu C, Huntzicker E, French D, et al. Intrahepatic aggregates of $\mathrm{T}$ cell and $\mathrm{B}$ cells are associated with the antiviral response to toll-like receptor 7 agonist GS-9620. Submitted.

[23] Belloni L, Allweiss L, Guerrieri F, Pediconi N, Volz T, Pollicino T, et al. IFNalpha inhibits HBV transcription and replication in cell culture and in humanized mice by targeting the epigenetic regulation of the nuclear cccDNA minichromosome. J Clin Invest 2012;122:529-537.

[24] Tropberger P, Mercier A, Robinson M, Zhong W, Ganem DE, Holdorf M. Mapping of histone modifications in episomal HBV cccDNA uncovers an unusual 
chromatin organization amenable to epigenetic manipulation. Proc Natl Acad Sci U S A 2015;112:E5715-5724.

[25] Riviere L, Gerossier L, Ducroux A, Dion S, Deng Q, Michel ML, et al. HBx relieves chromatin-mediated transcriptional repression of hepatitis $\mathrm{B}$ viral cccDNA involving SETDB1 histone methyltransferase. J Hepatol 2015;63:1093-1102.

[26] Wieland S, Thimme R, Purcell RH, Chisari FV. Genomic analysis of the host response to hepatitis B virus infection. Proc Natl Acad Sci U S A 2004;101:6669-6674.

[27] Fisicaro P, Valdatta C, Massari M, Loggi E, Ravanetti L, Urbani S, et al. Combined blockade of programmed death-1 and activation of CD137 increase responses of human liver T cells against HBV, but not HCV. Gastroenterology 2012;143:15761585 .

[28] Cheng X, Xia Y, Serti E, Block PD, Chung M, Chayama K, et al. Hepatitis B virus evades innate immunity of hepatocytes but activates macrophages during infection. Hepatology 2017.

[29] Thomsen MK, Nandakumar R, Stadler D, Malo A, Valls RM, Wang F, et al. Lack of immunological DNA sensing in hepatocytes facilitates hepatitis B virus infection. Hepatology 2016;64:746-759.

[30] Fletcher SP, Chin DJ, Cheng DT, Ravindran P, Bitter H, Gruenbaum L, et al. Identification of an intrahepatic transcriptional signature associated with self-limiting infection in the woodchuck model of hepatitis B. Hepatology 2013;57:13-22.

[31] Dunn C, Peppa D, Khanna P, Nebbia G, Jones M, Brendish N, et al. Temporal analysis of early immune responses in patients with acute hepatitis B virus infection. Gastroenterology 2009;137:1289-1300.

[32] Luangsay S, Gruffaz M, Isorce N, Testoni B, Michelet M, Faure-Dupuy S, et al. Early inhibition of hepatocyte innate responses by hepatitis B virus. J Hepatol 2015;63:1314-1322. 


\section{FIGURE LEGENDS}

Fig. 1. GS-9620-induced cytokines inhibit HBV DNA, RNA and antigen levels in HBV-infected PHH and dHepaRG cells. (A-E) HBV-infected PHH ( $\mathrm{n}=3$ donors) were treated with Mock-CM, GS-9620-CM or $100 \mathrm{IU} / \mathrm{mL}$ IFN- $\alpha$ from day 3-20, day 3-13 or day 13-20 post-infection. Plots show intracellular HBV RNA, extracellular HBV DNA, $\mathrm{HBeAg}$ and $\mathrm{HBsAg}$, as well as cell viability at day 20 post-infection. Data are expressed as a percentage of the no treatment (Tx) control; bar height indicates the mean and the error bars represent the SEM. (F) HBV-infected dHepaRG cells were treated with MockCM or GS-9620-CM from day 7-17 post-infection. Plot shows intracellular HBV DNA and extracellular $\mathrm{HBeAg}$ and $\mathrm{HBsAg}$ levels, as well as cell viability at day 17 postinfection. Data are expressed as a percentage of the no treatment control; bar height indicates the mean of $\mathrm{n}=2$ independent experiments and the error bars represent the SEM. Fresh media with CM or IFN- $\alpha$ was added every 2-4 days during the treatment phase of these studies. Statistical significance relative to the no treatment control was calculated with log-transformed values by one-way ANOVA with Dunnett's multiple comparison correction. $* \mathrm{p}<0.05, * * \mathrm{p}<0.01, * * * \mathrm{p}<0.0001$, ns: not significant $(\mathrm{p}>0.05)$.

Fig. 2. GS-9620-induced cytokines do not alter cccDNA levels in HBV-infected PHH and dHepaRG cells. HBV-infected PHH ( $\mathrm{n}=2$ donors) and $\mathrm{dHepaRG}$ were treated with Mock-CM or GS-9620-CM from day 3-13 post-infection (PHH) or day 7-17 postinfection (dHepaRG). HBV cccDNA was measured by (A) qPCR and (B) Southern Blot at day $13(\mathrm{PHH})$ or day 17 (dHepaRG) post-infection. HBeAg was also measured at day 
$13(\mathrm{PHH})$ or day 17 (dHepaRG) post-infection. The qPCR and HBeAg data are expressed as a percentage of the no treatment (Tx) control; bar height indicates the mean and the error bars represent the SEM. Statistical significance relative to the no treatment control was calculated with log-transformed values by one-way ANOVA with Dunnett's multiple comparison correction.

Fig. 3. Antiviral activity of GS-9620-induced cytokines in $\mathrm{HBV}$-infected $\mathrm{PHH}$ is dependent on the type I IFN receptor. (A) Cytokine levels in Mock-CM and GS-9620CM (both at 1:100 dilution) from healthy human PBMCs ( $n=3$ donors). Fold-induction in GS-9620-CM relative to Mock-CM is shown above each cytokine. (B-C) HBV-infected PHH (single donor, $\mathrm{n}=3$ independent experiments) were treated with Mock-CM, GS9620-CM or $100 \mathrm{IU} / \mathrm{mL}$ IFN- $\alpha$ from day 3-13 post-infection in the presence of IFNARblocking or isotype control (Ctrl) antibodies (Ab). (B) qRT-PCR data at day 13 postinfection expressed as fold-change relative to the no treatment control. (C) HBeAg levels at day 13 post-infection expressed as a percentage of the no treatment (Tx) control. (D) PHH ( $n=2$ donors) were infected with HBV, transfected with silFNAR1 (IFNAR) or siControl (Ctrl) 2 days post-infection and then treated with Mock-CM or GS-9620-CM from day 3-13 post-infection. qRT-PCR data at day 13 post-infection is shown; data is expressed as a percentage of Mock-CM. (E) HBeAg levels at day 13 post-infection for the study described in (D); data is expressed as a percentage of Mock-CM. For all plots, bar height indicates the mean and the error bars represent the SEM. Statistical significance relative to the no treatment control $(B, C)$ or $\operatorname{Mock-CM}(\mathrm{D}, \mathrm{E})$ was 
calculated with log-transformed values by one-way ANOVA with Dunnett's multiple comparison correction.

Fig. 4. Established HBV infection does not significantly alter the transcriptional response to GS-9620-induced cytokines. (A) Schematic summarizing the design of the study with two independent PHH donors. d: day, h: hour. (B) Immunofluorescence staining of HBV core (red) and nuclei (blue) at day 15 post-infection with HBV (+) or mock (-). (C) Number of differentially expressed genes (DEGs) for GS-9620-CM relative to time-matched Mock-CM (red, over-expressed; blue, under-expressed). (D) Correlation analyses of the transcriptional response to GS-9620-CM by Pearson correlation. The top two plots display $\log _{2}$-fold change values at 8 hours post-treatment with GS-9620-CM relative to time-matched Mock-CM for each $\mathrm{PHH}$ donor in $\mathrm{HBV}$-infected (+HBV) and mock-infected (-HBV) cells. The bottom plot displays $\log _{2}$ fold-change values at 8 hours post-treatment with GS-9620-CM relative to time-matched mock-CM for each donor in HBV-infected cells.

Fig. 5. GS-9620-induced cytokines stimulate an IFN response in PHH which is not significantly altered by $\mathrm{HBV}$ infection. Set-up described in Fig. 4A. (A) The top canonical pathways induced in $\mathrm{HBV}$-infected $\mathrm{PHH}$ (both donors) at 8 hours posttreatment with GS-9620-CM relative to time-matched Mock-CM were identified by Ingenuity Pathway analysis (IPA). The $-\log (p$-value) for $p=0.05$ and $p=0.01$ significance levels are indicated. The complete DEG list is provided in Supplementary Table 5. (B) Expression of ISGs significantly modulated by GS-9620-CM plotted by mean $\log _{2}$ fold- 
change relative to time-matched mock-CM control for each $\mathrm{PHH}$ donor. Rows represent individual genes; over-expression (red) and under-expression (blue) indicated by scale bars for $\log _{2}$ fold change values. The ISG DEG list is provided in Supplementary Table 6. (C) RNA-Seq data for select ISGs at 8 hours post-treatment with GS-9620-CM relative to time-matched mock-CM for each donor. False discovery rate (FDR) comparing GS9620-CM modulated expression change between mock-infected and HBV-infected PHH is displayed for each gene. Comparison data for all ISGs displayed in (B) is provided in Supplementary Table 7.

Fig. 6. Established HBV infection does not significantly modulate the transcriptional response to various innate immune stimuli. (A) Schematic summarizing the design of the study with three independent PHH donors treated with Mock-CM, GS-9620-CM, 100 IU/mL IFN- $\alpha, 10 \mu \mathrm{g} / \mathrm{mL}$ poly(I:C) or $10 \mathrm{HAU} / \mathrm{mL}$ Sendai virus (SeV). d: day, h: hour. (B) Immunofluorescence staining of $\mathrm{HBV}$ core (red) and nuclei (blue) at day 13 postinfection with HBV (+) or mock (-). (C) qRT-PCR data expressed as fold-change relative to no treatment control in mock-infected $\mathrm{PHH}$ for each donor. Bar height indicates mean and error bars represent the SEM. The symbols immediately above the bars denote the level of statistical significance relative to the no treatment control in mock-infected PHH: $* * \mathrm{p}<0.01 ; * * * \mathrm{p}<0.001$; ns: not significant $(\mathrm{p}>0.05)$. Statistical significance was calculated with log-transformed values by two-way ANOVA with Dunnett's multiple comparison correction. The p-values above the horizontal lines indicate the level of statistical significance between mock-infected and $\mathrm{HBV}$-infected $\mathrm{PHH}$ for each 
treatment. Statistical significance was calculated with log-transformed values by unpaired t-test.

\section{Fig. 7. GS-9620-induced cytokines enhance presentation of an immunodominant} HBsAg peptide by HBV-infected PHH. (A) Representative FACS profiles of T2 cells pulsed with $100 \mu \mathrm{g} / \mathrm{mL}$ of the indicated peptide and stained with the indicated antibody. (B) Representative confocal microscopy images of T2 cells pulsed with the indicated peptide and stained (red) with either the Env183-191 mAb (Env183 mAb) or an isotype control (ctrl). Nuclei were stained with DAPI (blue). The scale bar represents $10 \mu \mathrm{m}$. The data in (A) and (B) are representative of at least two independent experiments. (C) HBVinfected PHH (n=2 HLA-A0201 ${ }^{+}$donors) were treated with Mock-CM, GS-9620-CM or

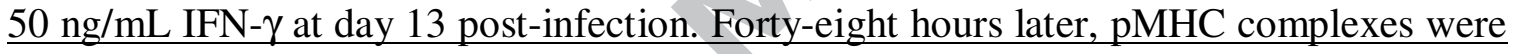
detected by staining with the Env183-191 mAb (red). Nuclei were stained with DAPI (blue). Representative confocal microscopy images from a single PHH donor are shown. The scale bar represents $10 \mu \mathrm{m}$. Quantitation of the number of pMHC complexes in each $\underline{\mathrm{PHH} \text { donor per treatment condition is displayed below the representative images. The bar }}$ height indicates the mean and error bars represent the SEM; at least 20 nuclei were analyzed per condition in each donor. The dotted lines indicate the level of background $\underline{\text { staining in uninfected PHH treated with IFN- } \gamma \text {. Statistical significance relative to the no }}$ treatment (Tx) control was calculated with log-transformed values by one-way ANOVA with Dunnett's multiple comparison correction. 
A
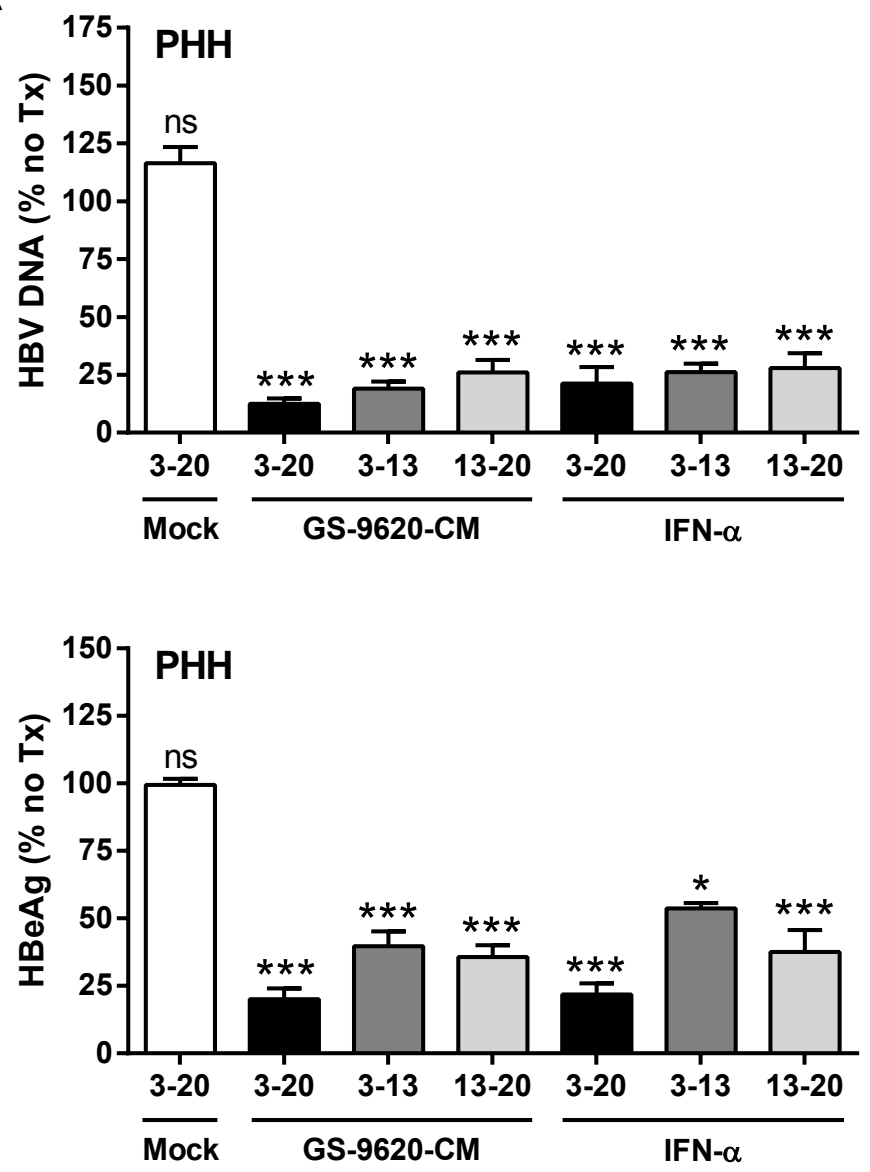

E

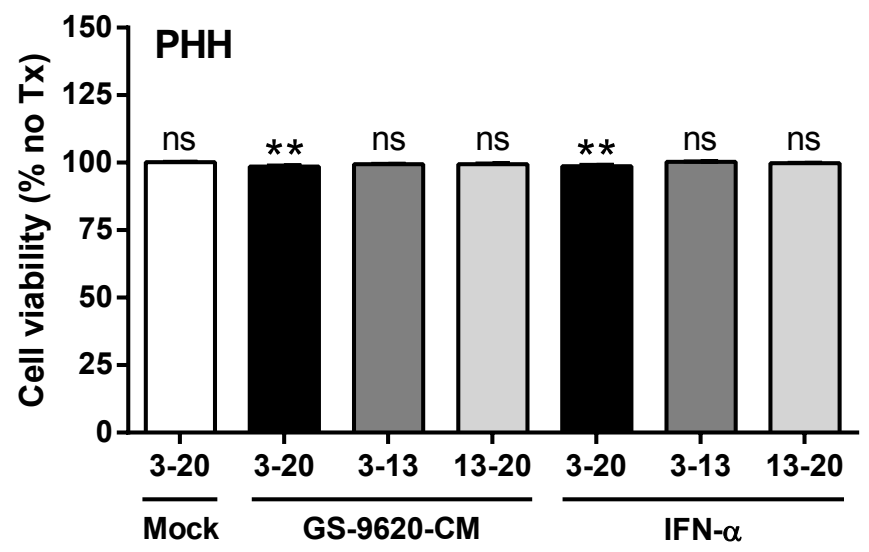

B

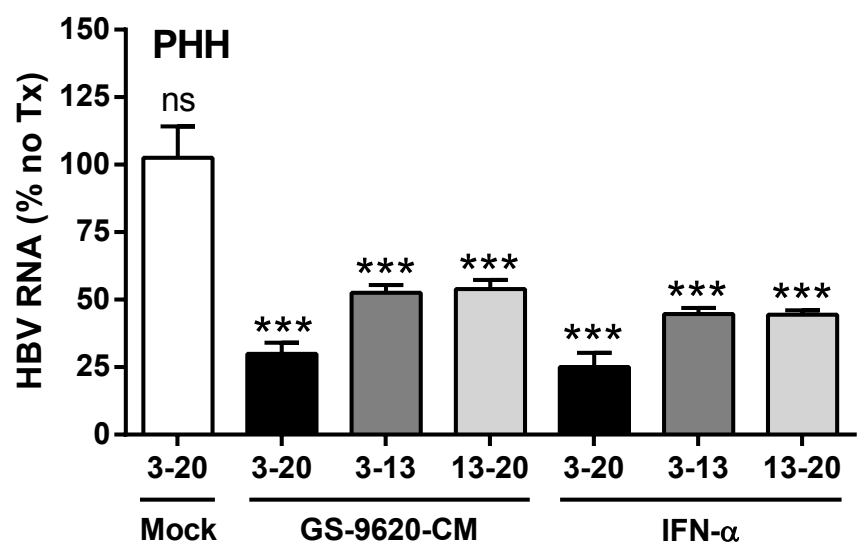

D

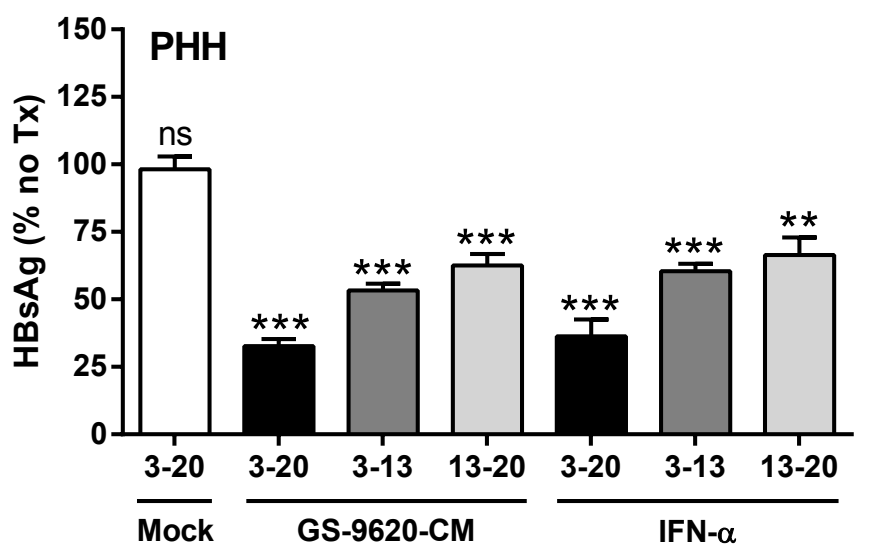

F

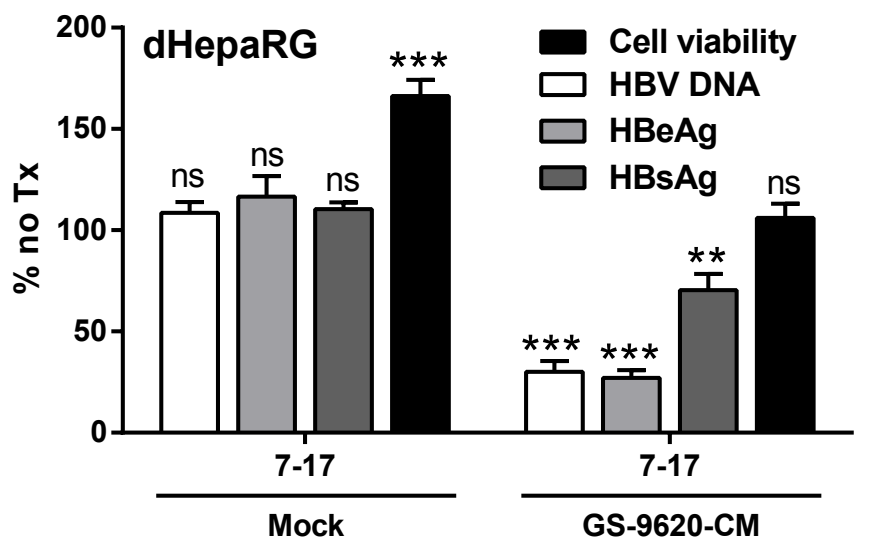


A
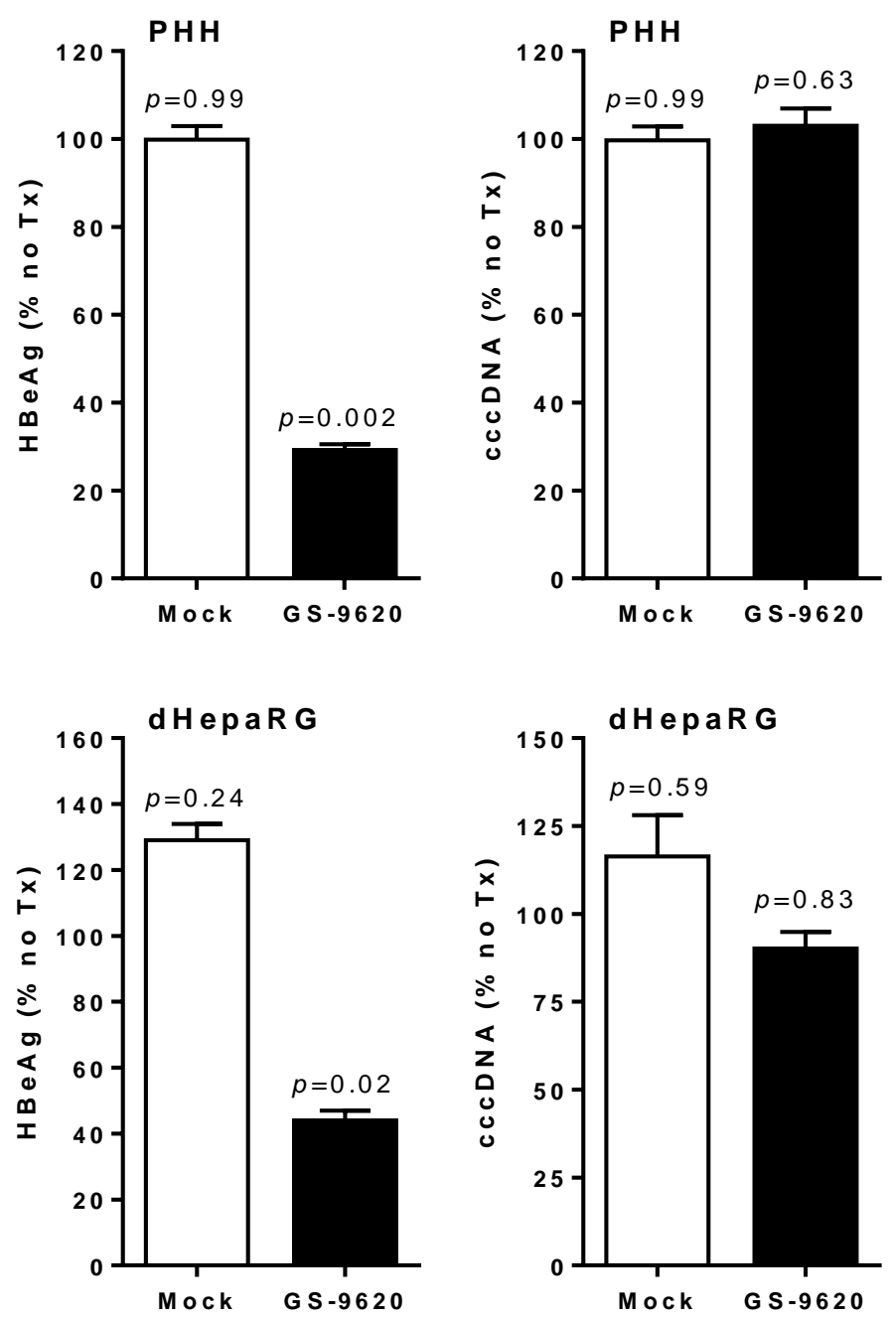

B

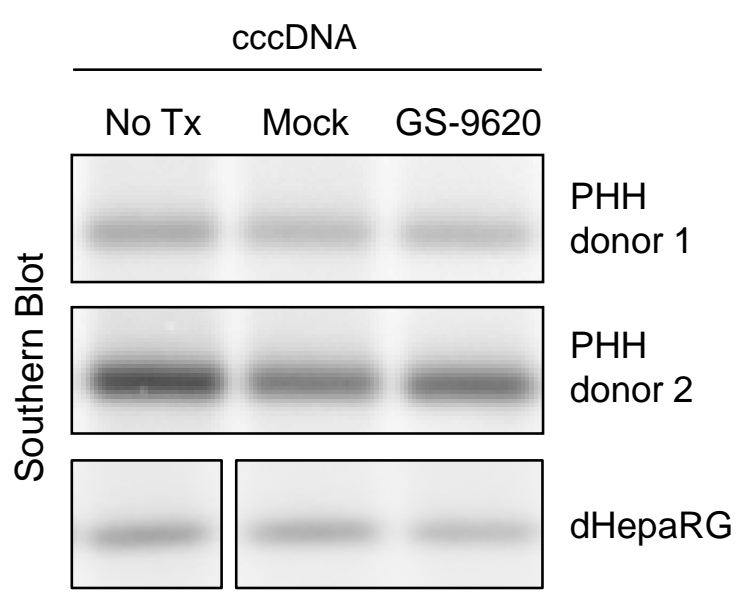


Figure 3

A

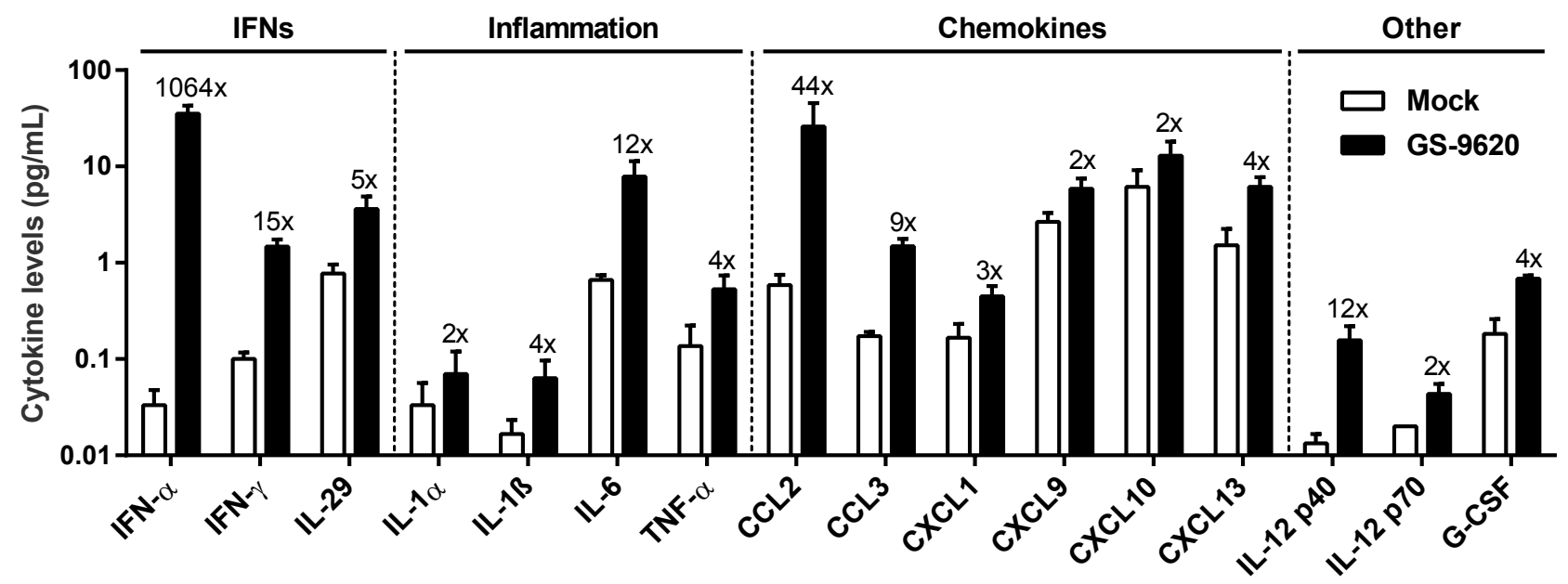

B
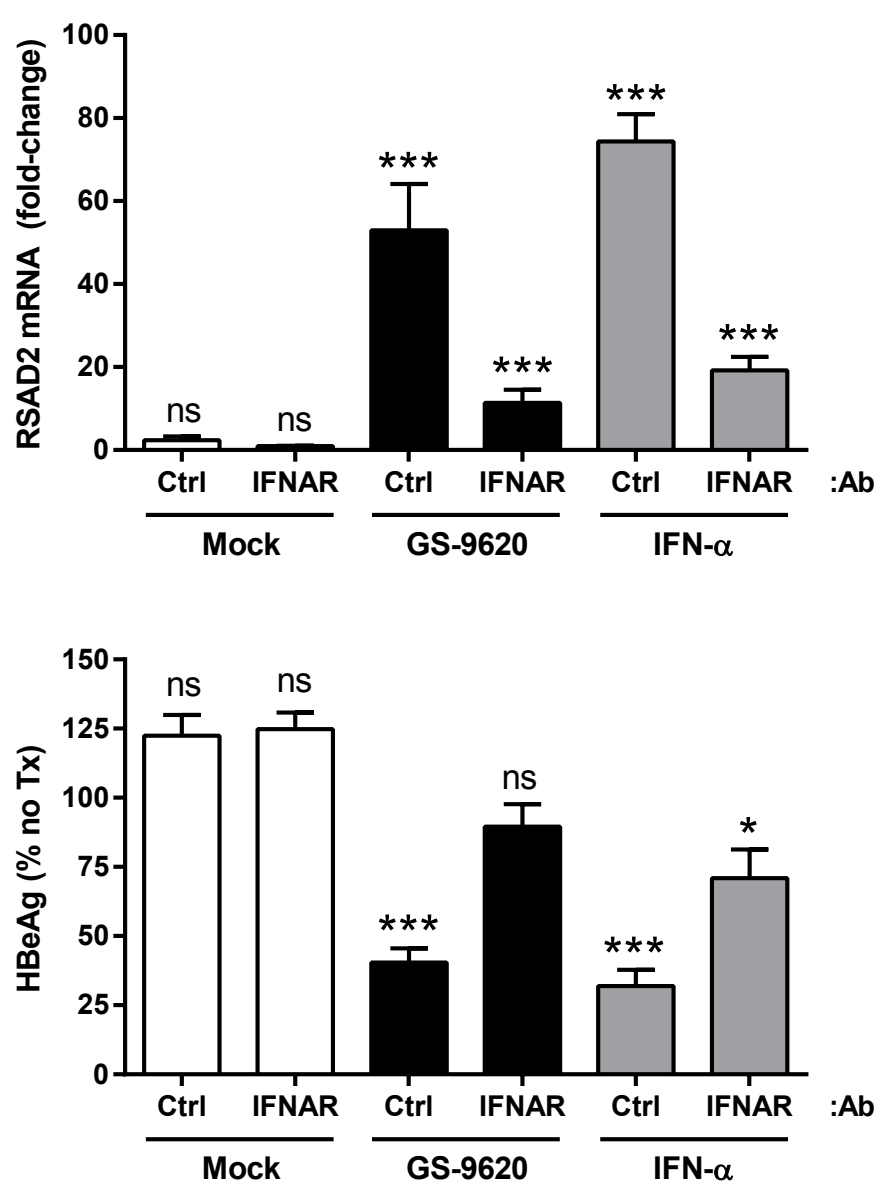

D

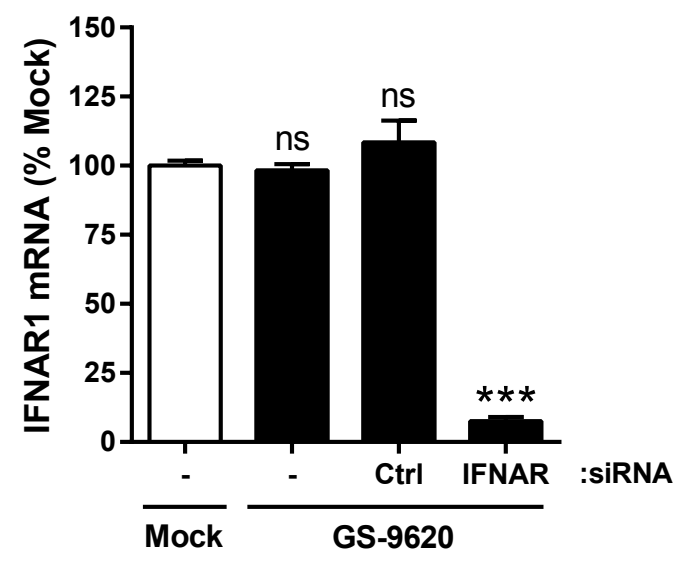

E

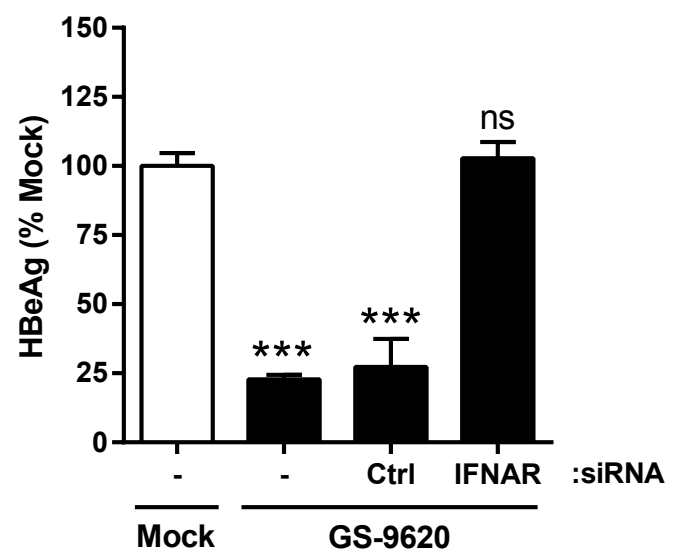


Figure 4

A

B

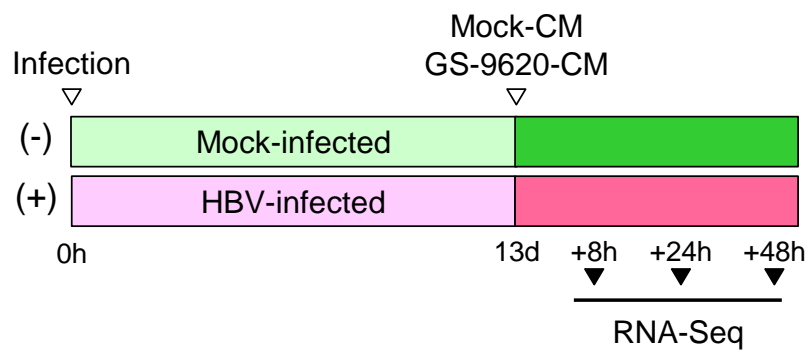

Donor 1

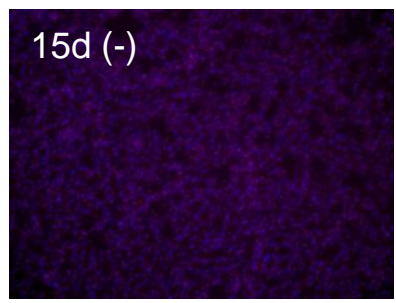

Donor 2
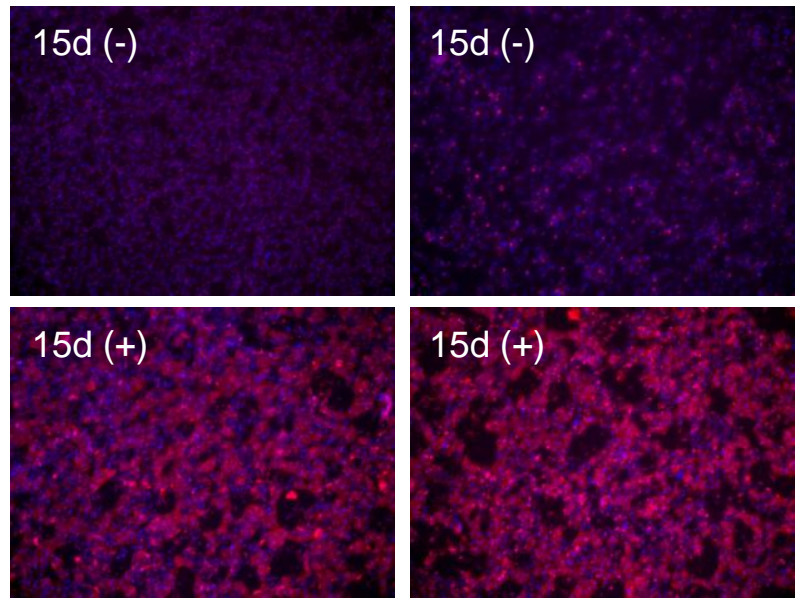

C

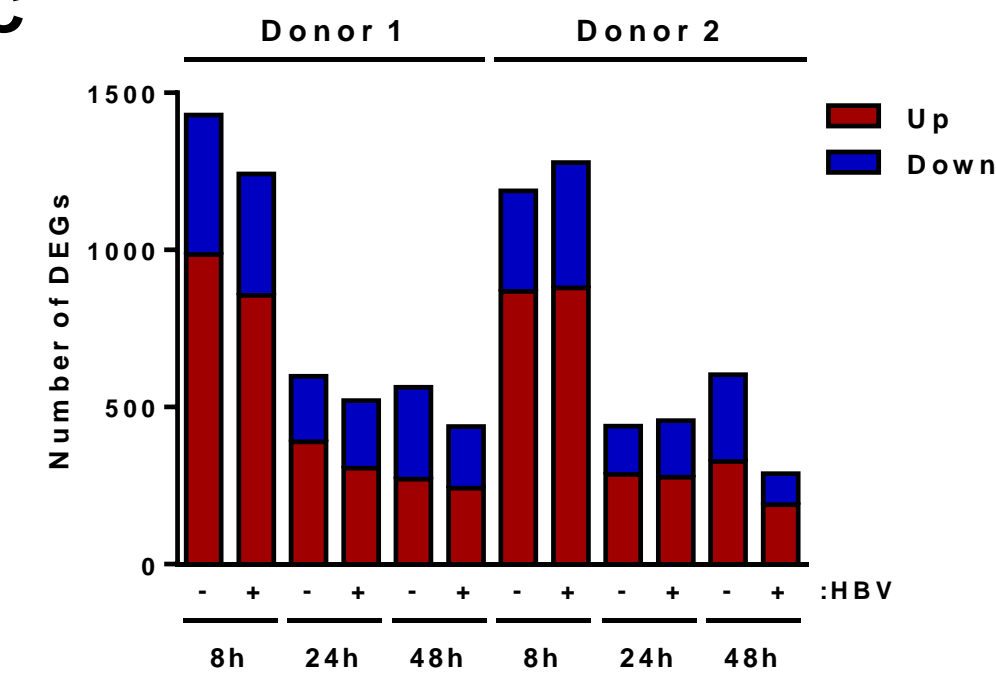

Mock-CM

RNA-Seq

Donor 2
D
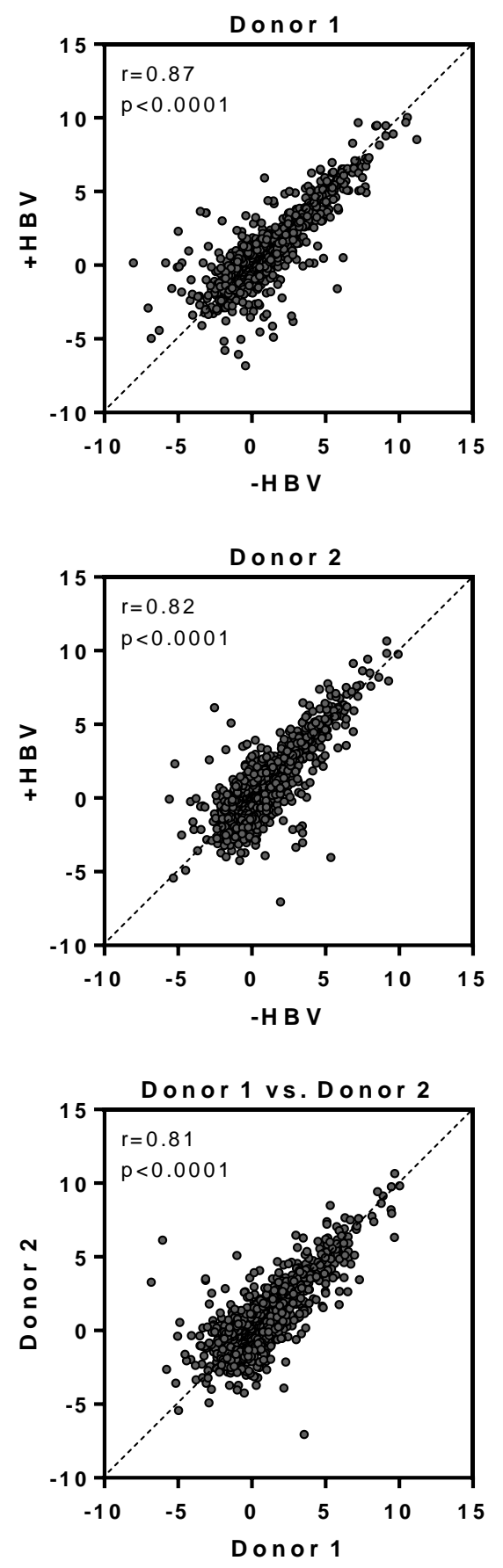

D 
Figure 5

A
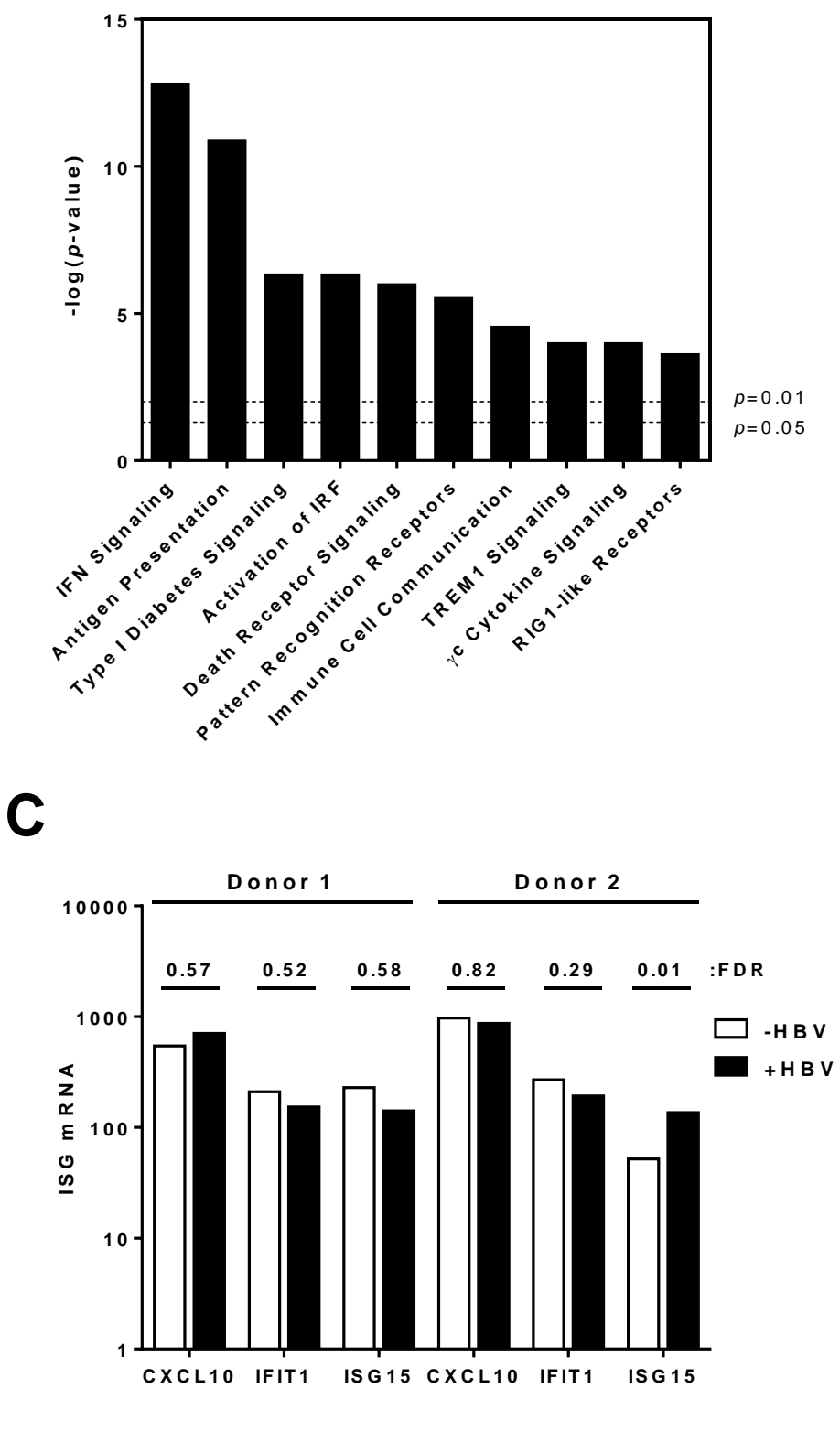

C

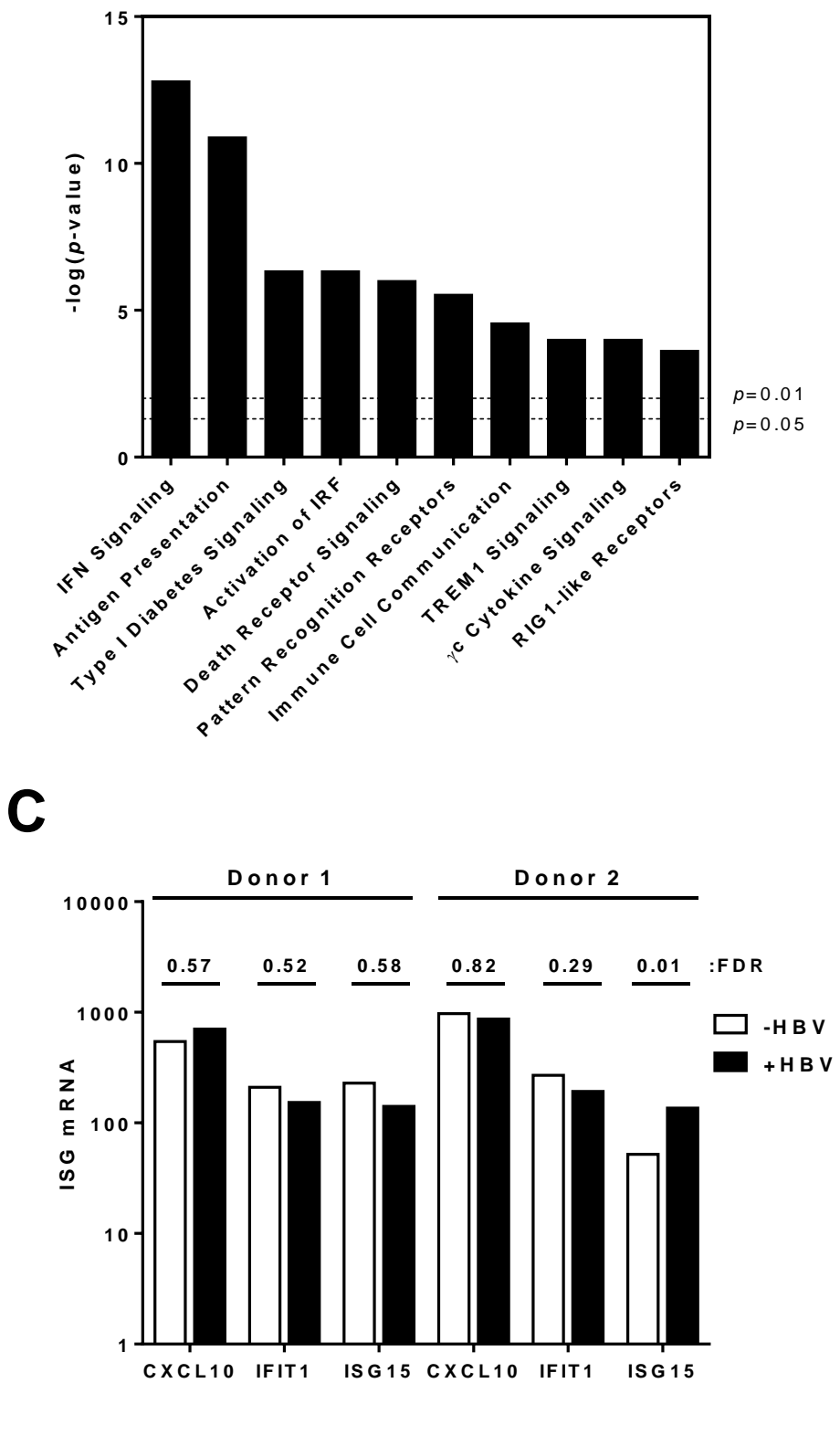

B

$\frac{\text { Donor } 1}{\frac{8 \mathrm{~h}}{-+} \frac{24 \mathrm{~h}}{-+} \frac{48 \mathrm{~h}}{-+} \frac{\text { Dh }}{-+} \frac{24 \mathrm{~h}}{-+} \frac{48 \mathrm{~h}}{-+}}$ :Time

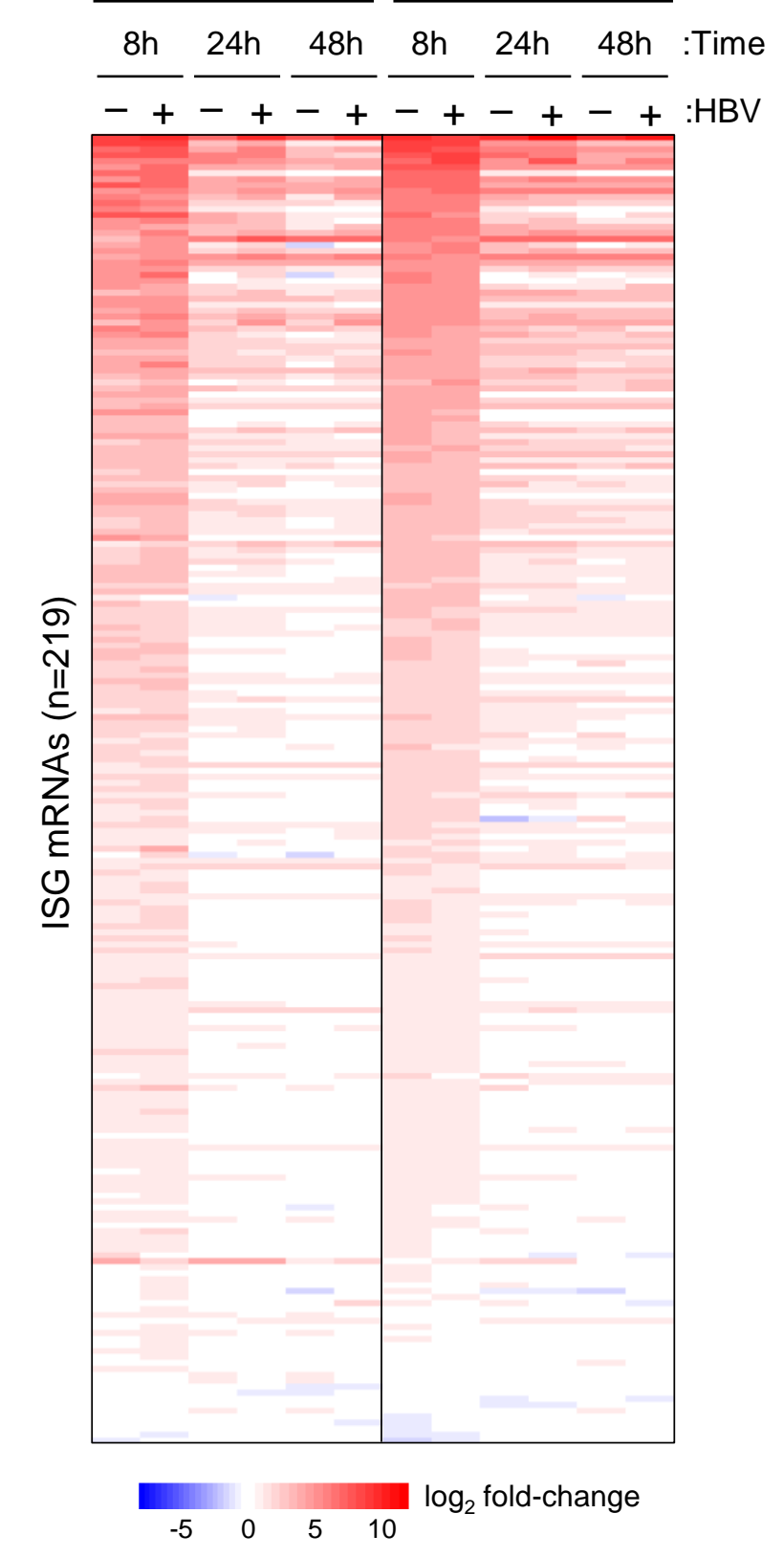


A Infection Mock-CM, GS-9620-CM

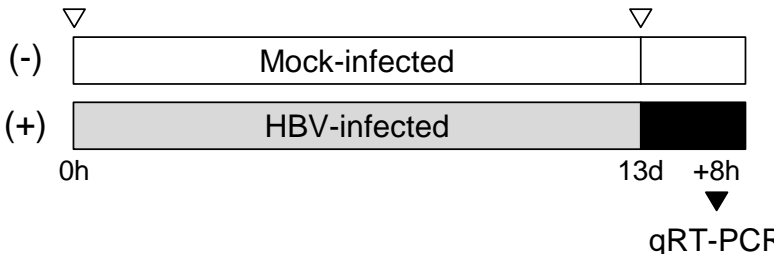

B Donor 1 Donor 2
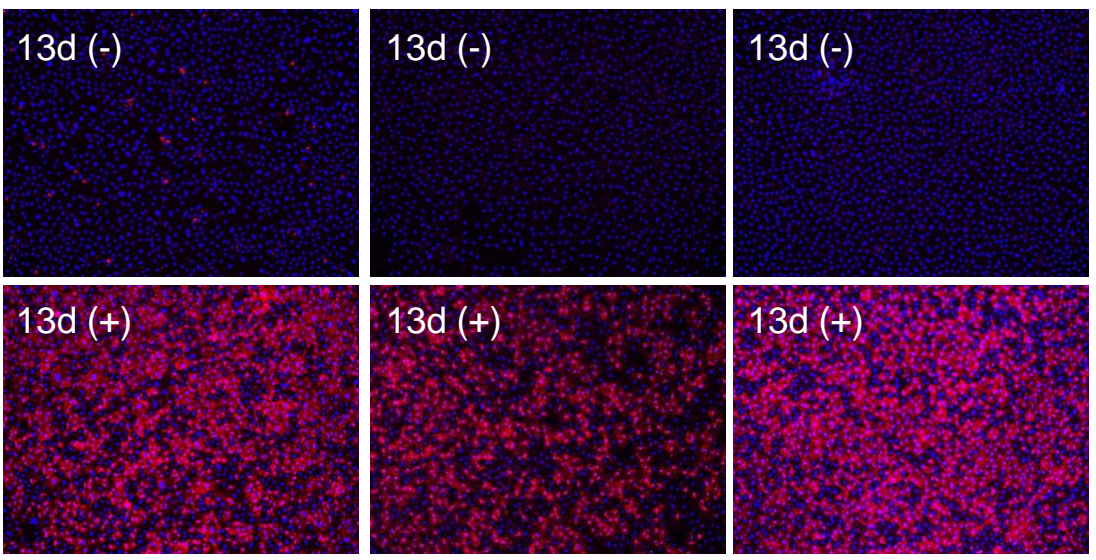

C
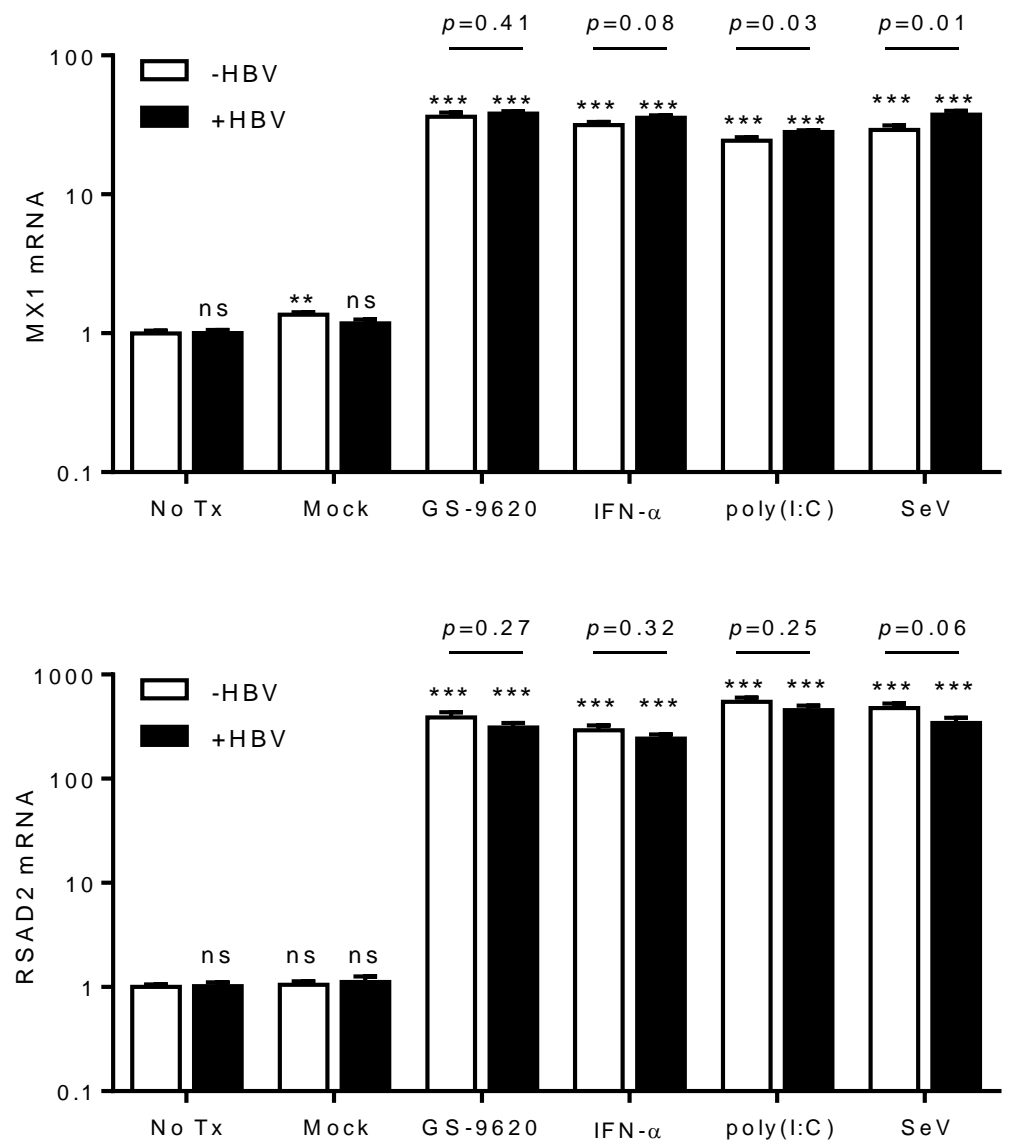
A

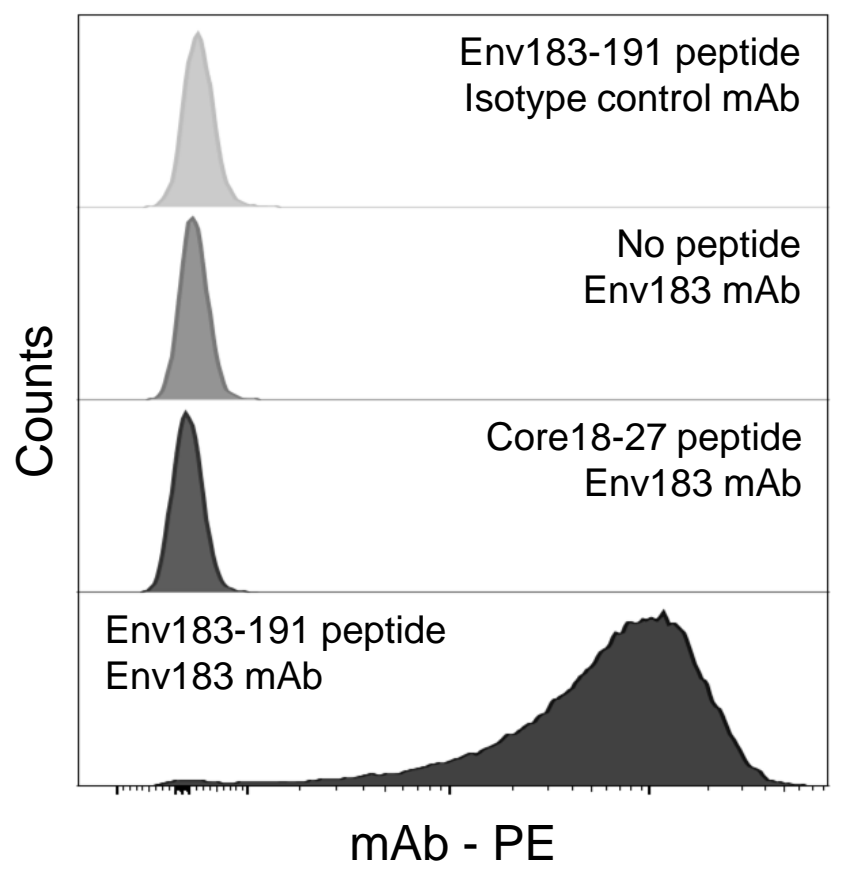

B

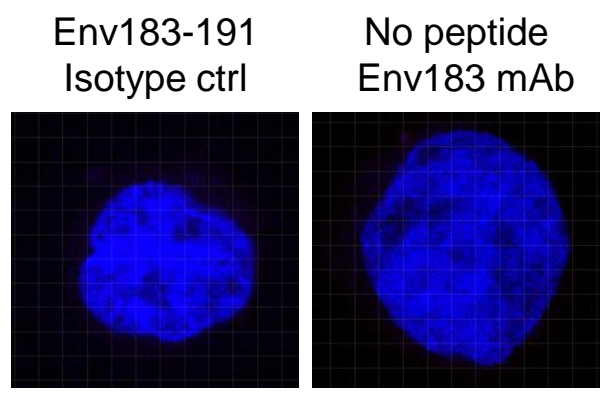

Core18-27

Env183-191

Env183 mAb

Env183 mAb
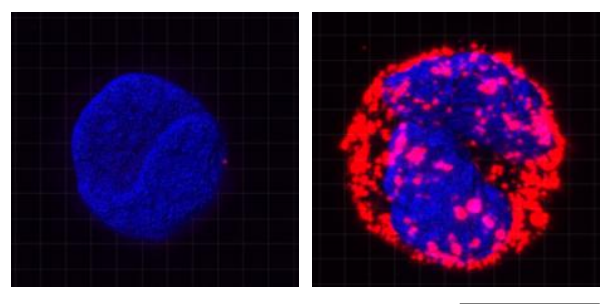

C
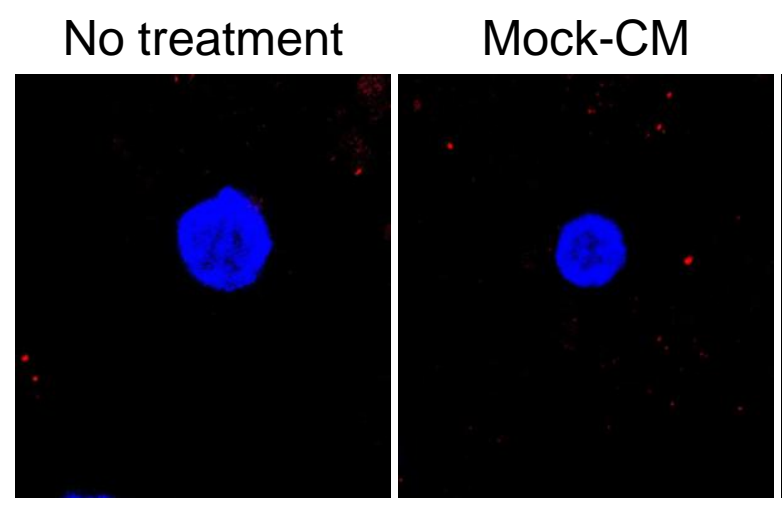

GS-9620-CM
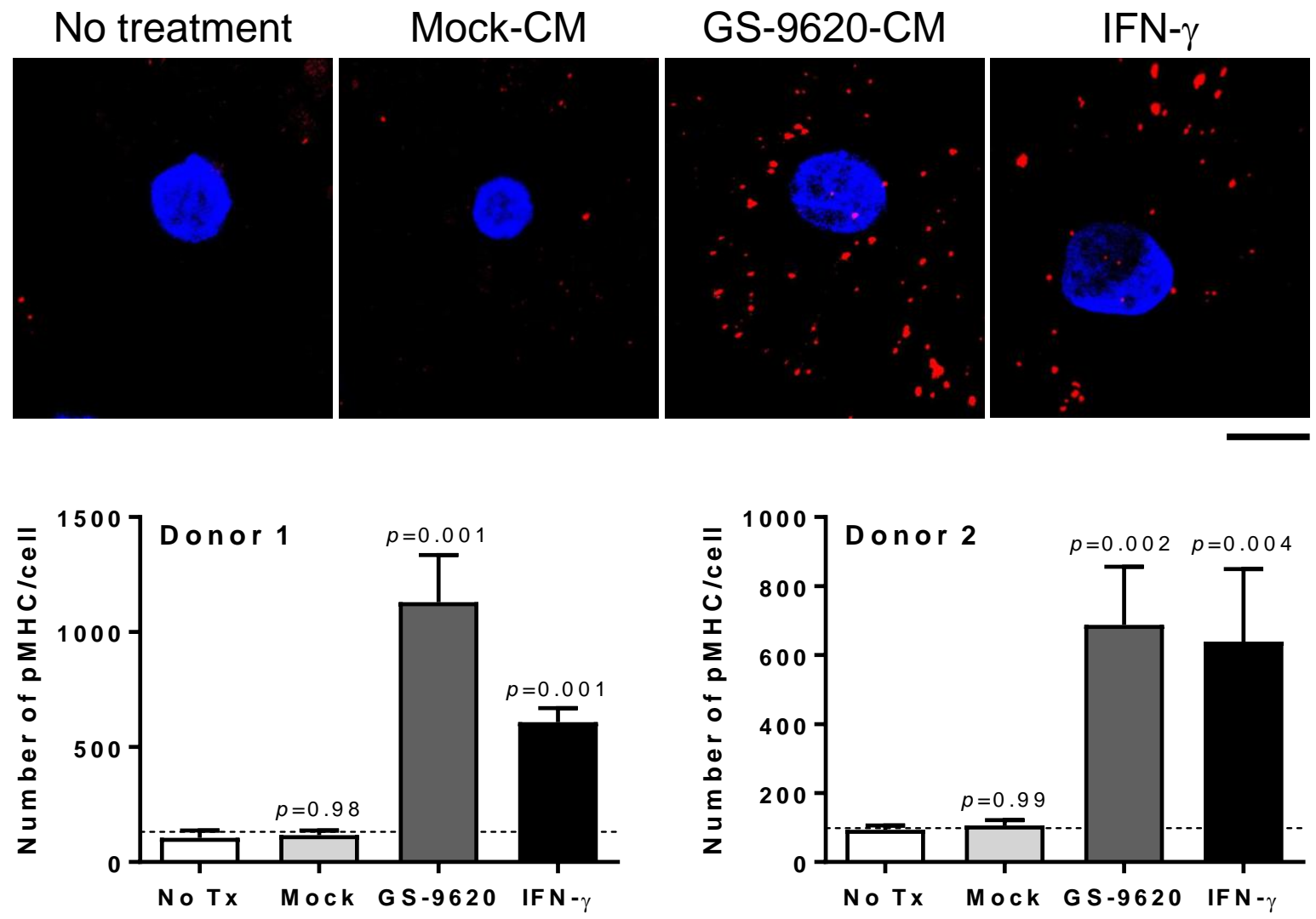
- GS-9620 has no direct antiviral activity against HBV

- Type I IFN induced by GS-9620 durably suppresses HBV without reducing cccDNA levels

- GS-9620-induced cytokines enhance HBV antigen presentation

- Established HBV infection does not modulate innate immune sensing or signaling 
CD8+ T CELL

GS-9620-induced type I IFN

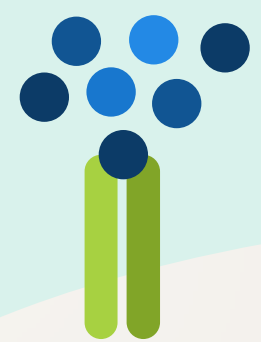

IFNAR1/2

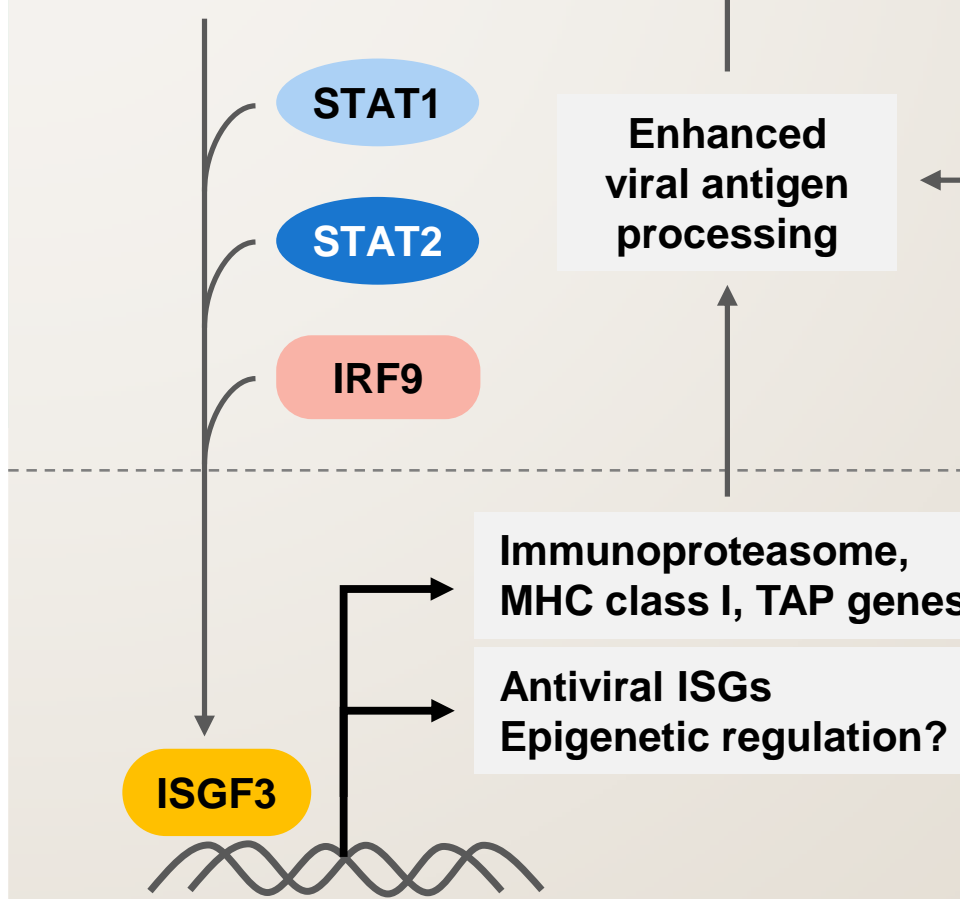

Host genome

\section{MHC class I}

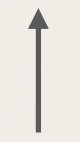

TCR

Enhanced $\mathrm{CD} 8+\mathrm{T}$ cell recognition of HBV-infected hepatocytes?

Altered viral antigen

presentation

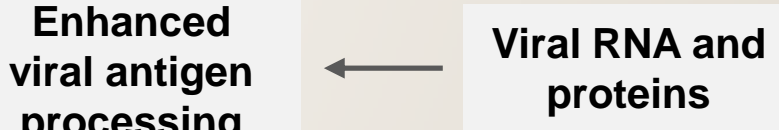

HBV-INFECTED HEPATOCYTE 